
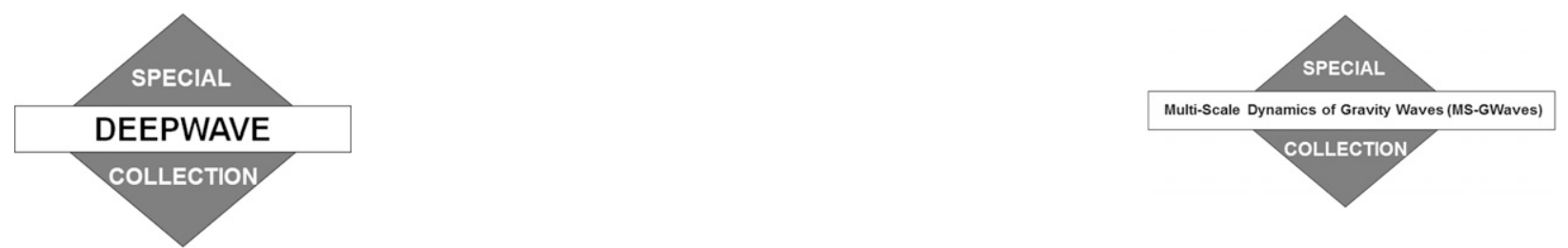

\title{
๖Nonlinear Simulations of Gravity Wave Tunneling and Breaking over Auckland Island
}

\author{
TYler MiXA, ${ }^{\mathrm{a}}$ ANDREAS DÖRNBRACK, ${ }^{\mathrm{a}}$ AND MARKus RAPP ${ }^{\mathrm{a}}$ \\ ${ }^{a}$ Deutsches Zentrum für Luft- und Raumfahrt, Institut für Physik der Atmosphäre, Weßling, Germany
}

(Manuscript received 30 July 2020, in final form 18 December 2020)

\begin{abstract}
Horizontally dispersing gravity waves with horizontal wavelengths of $30-40 \mathrm{~km}$ were observed at mesospheric altitudes over Auckland Island by the airborne advanced mesospheric temperature mapper during a Deep Propagating Gravity Wave Experiment (DEEPWAVE) research flight on 14 July 2014. A 3D nonlinear compressible model is used to determine which propagation conditions enabled gravity wave penetration into the mesosphere and how the resulting instability characteristics led to widespread momentum deposition. Results indicate that linear tunneling through the polar night jet enabled quick gravity wave propagation from the surface up to the mesopause, while subsequent instability processes reveal large rolls that formed in the negative shear above the jet maximum and led to significant momentum deposition as they descended. This study suggests that gravity wave tunneling is a viable source for this case and other deep propagation events reaching the mesosphere and lower thermosphere.
\end{abstract}

KEYWORDS: Gravity waves; Waves, atmospheric; Numerical analysis/modeling; Nonlinear models

\section{Introduction}

Large-amplitude gravity wave oscillations were observed directly above and in the lee of the Southern Ocean's Auckland Island $\left(50.8^{\circ} \mathrm{S}, 166.1^{\circ} \mathrm{E}\right)$ within the mesospheric airglow and sodium layers at $\sim 78-83 \mathrm{~km}$ altitudes during research flight RF23 of the Deep Propagating Gravity Wave Experiment (DEEPWAVE) (Fritts et al. 2016; Pautet et al. 2016). Remarkable for these altitudes, the gravity wave phase lines spread horizontally in a pattern common to the lower troposphere (Gjevik and Marthinsen 1978; Sharman and Wurtele 1983; Mitchell et al. 1990): they resemble transverse phase lines of trapped mountain waves in the lee of three-dimensional obstacles (Smith 1989; Sharman and Wurtele 2004), suggesting that the observed mesospheric temperature perturbations originated from the flow across Auckland Island. This hypothesis was supported by the sustained appearance of the observed gravity waves in the hydroxyl $(\mathrm{OH})$ brightness and retrieved temperature perturbations from the Advanced Mesospheric Temperature Mapper (AMTM), which remained stationary for several hours (Pautet et al. 2016). Simultaneous lidar measurements of sodium mixing ratios in the mesosphere and lower thermosphere (MLT) indicate peak gravity wave amplitudes of $\approx \pm 10 \mathrm{~K}$ at $z \approx 83 \mathrm{~km}$ and $\lambda_{x} \approx 40 \mathrm{~km}$. Later flight legs show strong indications of gravity wave breaking, with

¿ Denotes content that is immediately available upon publication as open access.

Corresponding author: Tyler Mixa, t.mixa@gats-inc.com apparent vortex ring formation and momentum fluxes estimated over $320 \mathrm{~m}^{2} \mathrm{~s}^{-2}$ (Pautet et al. 2016).

Eckermann et al. (2016) investigated how these orographic gravity waves could reach the MLT with relatively small, linear amplitudes. They reproduced key aspects of the observed MLT wave field by applying a three-dimensional Fourier ray model constrained by high-altitude reanalysis data from the Navy Global Environmental Model (NAVGEM) (Hogan et al. 2014; Eckermann et al. 2018). Because their Fourier ray model could not account for nonlinear dynamics, the momentum flux and resulting gravity wave drag (GWD) were estimated from linear saturation theory and found to agree quantitatively with estimates from Pautet et al. (2016). A follow-on study by Broutman et al. (2017) computed stationary phase solutions from the same Fourier model, finding that nonhydrostatic gravity wave modes spread over a larger geographical region than the hydrostatic modes closer to Auckland Island. The reduced intrinsic phase speed of the hydrostatic waves allowed stronger horizontal advection of the wavenumber vector, producing an extended lee wave response similar to horizontal propagation over South Georgia Island simulated by Vosper (2015).

Eckermann et al. (2016) concluded that the gravity waves observed in the MLT were comprised of linear, nonhydrostatic gravity wave modes that remained stable up to $\sim 70-80 \mathrm{~km}$ altitudes and reached the MLT in $1.5-4 \mathrm{~h}$. The authors identified three stabilizing dynamical effects in their Fourier solutions that reduce the amplitude and keep the waves linear up to the MLT: (i) refraction by background winds, (ii) horizontal spreading of the wave field, and (iii) erosion of specific harmonics with intrinsic frequencies $\left(\omega_{i}\right)$ that experience directional critical levels $\left(\omega_{i} \rightarrow 0\right)$ and turning levels $\left(\omega_{i} \rightarrow N\right)$, where $N$ is the Brunt-Väisälä frequency. Some of these modes 

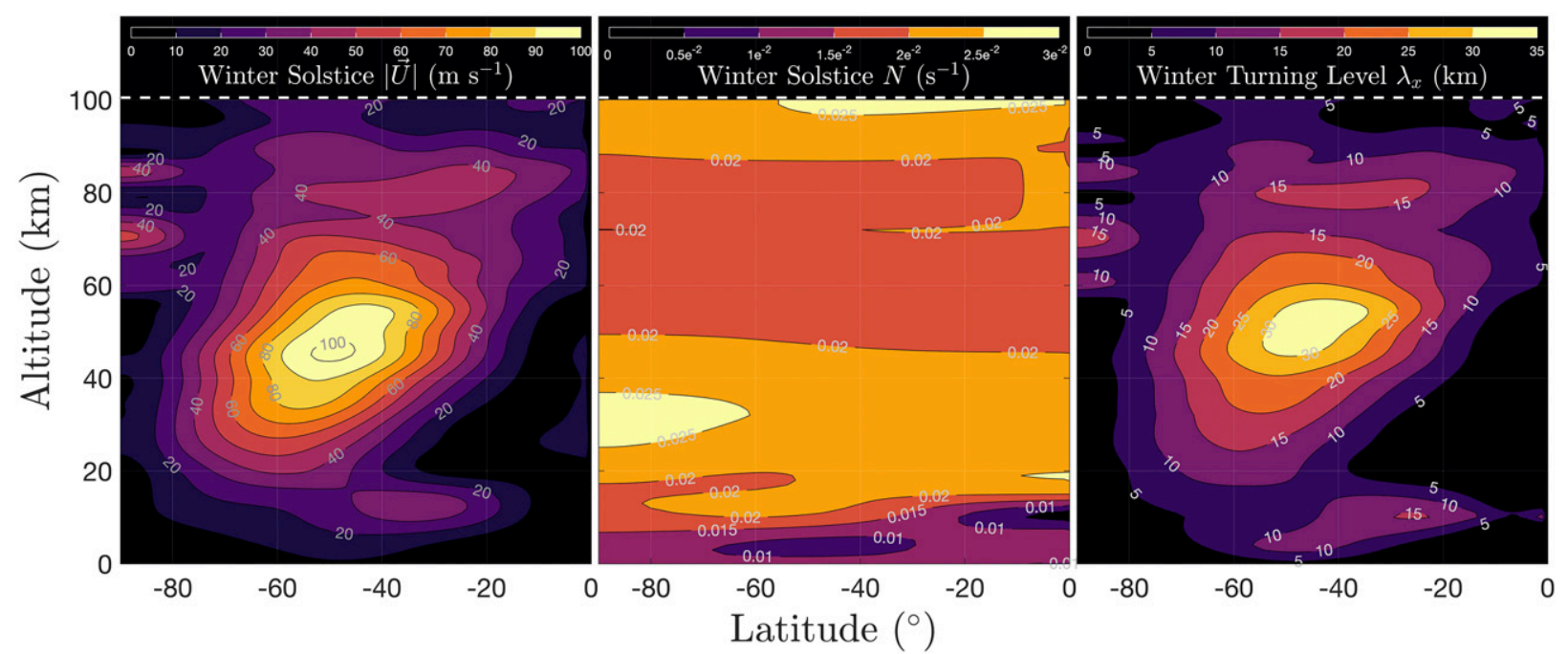

FIG. 1. Winter solstice climatology for (left) the magnitude of the zonal wind, taken from the Horizontal Wind Model (Drob et al. 2015); (center) the Brunt-Väisälä frequency, taken from the 2001 U.S. Naval Research Laboratory Mass Spectrometer and Incoherent Scatter Radar Exosphere (NRLMSISE-00; Picone et al. 2002); and (right) the distribution of turning levels for stationary gravity waves of varying zonal wavelengths $\lambda_{x}$.

removed at turning levels can exhibit partial transmission by tunneling, where gravity waves penetrate through a local evanescent $\left(\omega_{i} \geq N\right)$ region instead of fully reflecting. Broutman et al. (2009) demonstrated that the Fourier ray model can estimate the transmission of tunneling modes through linear interpolation over the evanescent region. However, Eckermann et al. (2016) did not include these tunneling transmission estimates, truncating the spectra to completely remove all evanescent modes at each turning level.

The evanescent modes removed from this analysis correspond to high-frequency, short-horizontal-wavelength $\left(\lambda_{x} \lesssim 30 \mathrm{~km}\right)$ components known as "roughness modes" (Smith and Kruse 2017), which can be generated by small-scale features of Auckland Island's topography. During the 2014 DEEPWAVE campaign, Smith and Kruse (2017) found that roughness modes $\left(\lambda_{x} \sim 8-40 \mathrm{~km}\right)$ contributed more than twice the momentum flux of longer terrain modes $\left(\lambda_{x} \sim 200-400 \mathrm{~km}\right)$ measured by in situ aircraft data over New Zealand. If roughness modes were evaluated for the Auckland Island case, these modes could quickly reach the MLT and contribute to the instability dynamics seen in the AMTM at later times.

Gravity waves with short horizontal wavelengths are generally not included in global circulation models due to their high resolution requirements and their limited influence according to linear gravity wave theory. Linear theory for stationary mountain waves predicts a cutoff wavelength of $\lambda_{x \text { cutoff }}=2 \pi u / N \geqslant 30-50 \mathrm{~km}$ inside the polar night jet (PNJ) (Schoeberl 1985). This cutoff suggests a widespread existence of turning levels for mountain waves with $\lambda_{x} \lesssim 30 \mathrm{~km}$ in the winter hemisphere, as shown in Fig. 1. Thus, the mountain wave response in the MLT depends on the horizontal wind and temperature fields through which they propagate and with which they often interact strongly (Bramberger et al. 2017; Kruse et al. 2016).
Many small, subantarctic islands are known to generate orographic gravity waves at or below the cutoff wavelengths predicted by linear theory. These sources produce enhanced gravity wave variances up to $50 \mathrm{~km}$ altitudes in the winter stratosphere, as seen in satellite measurements $(\mathrm{Wu}$ et al. 2006; Alexander et al. 2009; Alexander and Grimsdell 2013; Hoffmann et al. 2016; Hindley et al. 2019) and confirmed by high resolution numerical studies (Vosper 2015). The momentum flux contribution of these subgrid-scale gravity waves has been found to be climatologically significant from both small island orographic sources (Alexander and Grimsdell 2013; Plougonven et al. 2017) and nonorographic sources that include convection, frontogenesis, and spontaneous emission in rapidly evolving baroclinic systems (Hendricks et al. 2014; Plougonven et al. 2015).

Roughness mode gravity waves have also been observed propagating above the PNJ. Over a 3-yr study of gravity wave characteristics in the Arctic winter mesosphere, $\mathrm{Li}$ et al. (2020) observed numerous high-frequency (near- $N$ ), small-scale $\left(\lambda_{x}<20 \mathrm{~km}\right)$ gravity waves propagating into the wind at $\sim 87 \mathrm{~km}$ over northern Canada. In the Southern Hemisphere, the 2014 DEEPWAVE campaign revealed that a broad variety of small-scale $\left(\lambda_{x} \sim 10-40 \mathrm{~km}\right)$ gravity waves can reach the winter MLT (Pautet et al. 2019), where highfrequency gravity wave responses transported the most momentum and eastward (upwind) gravity wave propagation correlated with elevated tropospheric forcing below. Given that small-scale gravity waves have been observed above the PNJ in the winter MLT, and given that several orographic and nonorographic sources have been shown to generate these scales of mesospheric gravity wave responses, it is essential to evaluate how tropospheric gravity wave modes at or below the linear cutoff wavelength pass through the PNJ and determine how these gravity wave modes contribute to instability processes in the MLT region. 
Here, we apply the three-dimensional nonhydrostatic, compressible flow solver EULAG (Prusa et al. 2008) to investigate the deep vertical propagation of mountain waves from the flow across Auckland Island. The first goal of this study is to elaborate the role of wave tunneling through the PNJ for the Auckland Island case. The strong impinging winds across the remote localized topography together with the nearly zonally aligned PNJ make this laboratory-like case an ideal scenario to study both linear as well as nonlinear aspects of vertical mountain wave propagation, as all other wave sources can be excluded. This paper proposes tunneling through the PNJ as an enabler of high-frequency gravity wave penetration into the MLT, demonstrating this effect in a case study of the 2014 DEEPWAVE observations over Auckland Island.

Observational evidence for wave tunneling for a fluid at rest was presented by Sutherland and Yewchuck (2004). There, wave tunneling occurred through a well-mixed layer of strongly reduced Brunt-Väisälä frequency $N_{0}$ sandwiched between two wave regions of propagation with $N>N_{0}$. Sutherland and Yewchuck (2004) theoretical analysis of the laboratory experiment revealed that wave packets incident from below became evanescent in the uniformly stratified $N_{0}$ layer and were able to transmit wave energy upward, a process called wave tunneling in analogy to quantum mechanics where free electrons tunnel across a potential barrier. Tunneling through evanescent regions associated with the tropopause jet was reported by Eckermann et al. (2006a) for flow across Jan Mayen and Eckermann et al. (2006b) for flow across Scandinavia. Nault and Sutherland (2008) extended the theoretical analysis by Sutherland and Yewchuck (2004) and computed the transmission of smallamplitude internal gravity waves for an inviscid fluid having arbitrarily specified stratification and background velocity. They confirmed that internal waves could tunnel through an evanescent region near the tropopause and radiate into the stratosphere.

The results of our study demonstrate how tunneling enables quick propagation of small-scale gravity waves from a localized topographic source of only $20 \mathrm{~km}$ extent through the PNJ, radiating gravity waves deep into the mesosphere. These gravity waves generate a wide region of gravity wave breaking and instabilities resulting in peak GWD above the PNJ. The associated momentum deposition is comparable to the largest gravity wave momentum fluxes observed in the mesosphere.

The paper is organized as follows. In section 2 we first outline the numerical model as it is its first application to simulate a realistic case of deep wave propagation. Section 3 presents the numerical results. Concluding remarks are given in section 4 .

\section{Methodology}

\section{a. Numerical integrations}

The nonlinear numerical simulations were conducted with EULAG, the Eulerian/semi-Lagrangian fluid solver (Smolarkiewicz and Margolin 1997; Prusa et al. 2008). Here, the compressible Euler equations are integrated. The conservation laws for momentum, internal energy, and mass complemented by the ideal gas law are written in the form presented by Smolarkiewicz et al. (2014):

$$
\begin{aligned}
\frac{d \mathbf{u}}{d t} & =-c_{p} \theta \nabla \pi^{\prime}-\mathbf{g} \frac{\theta^{\prime}}{\theta_{e}}-\mathbf{f} \times\left(\mathbf{u}-\frac{\theta}{\theta_{e}} \mathbf{u}_{e}\right)-\alpha\left(\mathbf{u}-\mathbf{u}_{e}\right)+\mathbf{F}, \\
\frac{d \theta^{\prime}}{d t} & =-\mathbf{u} \cdot \nabla \theta_{e}-\beta \theta^{\prime} \\
\frac{d \rho}{d t} & =-\rho \nabla \cdot \mathbf{u} \\
\pi & =\left(\frac{R_{d}}{p_{0}} \rho \theta\right)^{R_{d} / c_{v}} .
\end{aligned}
$$

Here, vector $\mathbf{u}$ denotes the flow velocity, $\theta$ is the potential temperature, $\rho$ is the density, and $\pi \equiv\left(p / p_{0}\right)^{R_{d} / c_{p}}$ denotes the Exner function, with pressure $p$ and the constant reference pressure $p_{0}$. The specific heat at constant volume is related to the specific heat at constant pressure by $c_{v}=c_{p}-R_{d}$, where $R_{d}$ is the gas constant for dry air. Absolute temperature is computed by $T=\theta \pi$.

The magnitude of the gravitational acceleration is given by $\mathbf{g}=(0,0,-g)$. The Coriolis parameter is given as $\mathbf{f} \equiv 2 \boldsymbol{\Omega}$, where $\boldsymbol{\Omega}$ denotes a constant angular velocity of the rotating reference frame. The differential operators in Eqs. (1)-(3) are defined as $d / d t=\partial / \partial t+\mathbf{u} \cdot \nabla$.

Equation (2) is written in perturbation form, i.e., $\theta^{\prime}=\theta-\theta_{e}$; see Smolarkiewicz et al. (2019). To facilitate the numerical solution, these auxiliary ambient states, sometimes called environmental states $\left(\mathbf{u}_{e}, \theta_{e}\right)$, are assumed to be a known particular solution of the Eqs. (1) and (2). Generally, ambient states can be time dependent, e.g., prescribing tidal motions (WarnVarnas et al. 2007). In this paper, only $z$-dependent profiles $\left(u_{e}\right.$, $\left.v_{e}, \theta_{e}\right)$ are prescribed that automatically satisfy Eqs. (1) and (2).

The terms in Eqs. (1) and (2) involving $\alpha(\mathbf{x}, t)$ and $\beta(\mathbf{x}, t)$ represent forcings to attenuate the solution to the prescribed environmental profiles $\mathbf{u}_{e}$ and $\theta_{e}$. The forcing term $\mathbf{F}=\left(F_{x}, F_{y}\right.$, 0 ) in Eq. (1) denotes an additional acceleration to ramp up the flow; see next section.

The Eqs. (1)-(4) were implemented in the established soundproof model EULAG (Prusa et al. 2008) as outlined by Smolarkiewicz et al. (2014). There, governing equations are formulated in generalized time-dependent curvilinear coordinates to enable mesh adaptivity (Prusa and Smolarkiewicz 2003; Wedi and Smolarkiewicz 2004; Kühnlein et al. 2012; Smolarkiewicz and Charbonneau 2013) and continuous mappings of Earth's topography by using terrain-following coordinates (Gal-Chen and Somerville 1975; Clark 1977). Here, only stationary mesh adaptivity is used. In symbolic form, Eqs. (1)-(3) are written for the specific variables $\psi=\left(u, v, w, \theta^{\prime}, 1\right)$ as

$$
\frac{\partial \mathcal{G} \varrho \psi}{\partial t}+\nabla \cdot(\mathcal{G} \varrho \mathbf{v})=\mathcal{G} \varrho \mathcal{R}
$$

where $(u, v, w)$ are the Cartesian components of the velocity vector, $\varrho(\mathbf{x}, t)$ is the density in the coordinates $(\mathbf{x}, \mathrm{t})$ of the generalized frame, and $\mathcal{G}(\mathbf{x}, t)$ is the Jacobian of the transformation (Prusa and Smolarkiewicz 2003). As before, $\nabla \ldots$ denotes the scalar product of spatial derivatives with a vector, so $d / d t=\partial / \partial t+\mathbf{v} \cdot \nabla$, where the velocity $\mathbf{v}=\dot{x}$ is not necessarily the physical velocity $\mathbf{u}$. The function $\mathcal{R}$ comprises all forcings on the right-hand sides of Eqs. (1)-(3). The special case of $\psi=1$ and $\mathcal{R}=0$ leads to the generalized mass continuity equation. 
The numerical integration scheme is described in detail in Smolarkiewicz et al. (2014). It is based on nonoscillatory forward-in-time (NFT) differencing and can be summarized as

$$
\Psi_{i}^{n+1}=\mathcal{A}_{i}\left(\tilde{\Psi}^{n}, \mathbf{V}^{n+1 / 2}, \mathcal{G}^{n}, \mathcal{G}^{n+1}\right)+0.5 \delta t \mathcal{R}_{i}^{n+1},
$$

where the auxiliary field $\tilde{\Psi}^{n} \equiv \Psi^{n}+0.5 \delta t \mathcal{R}^{n}$ is transported. This difference to the advection of the specific variable $\Psi$ results from a rigorous truncation error analysis (Smolarkiewicz and Margolin 1997). The NFT scheme in (6) belongs to the class of second-order-accurate two-time-level algorithms that are built on nonlinear advection schemes (Smolarkiewicz and Margolin 1998). These schemes have the property to suppress and control numerical oscillations that are often found in higher-order linear schemes. Therefore, they can be used to conduct inviscid numerical integration of the Euler Eqs. (1)-(3) by means of the algorithm (6).

We present the results of two 3D EULAG simulations in this analysis. Simulation 1 was run at a baseline resolution with no wind ramp to evaluate the time evolution of the event and the stratospheric propagation characteristics. The results from simulation 1 are presented in sections $3 a$ and $3 b$. Simulation 2 doubled the baseline horizontal grid resolution and employed a gradual wind ramp (see, e.g., Lund et al. 2020) to prevent acoustic artifacts of an instantaneous wind initialization from influencing the instability characterization and momentum deposition at high amplitudes. The results of simulation 2 are presented in sections $3 \mathrm{c}$ and $3 \mathrm{~d}$. Descriptions of the model domain and the initial and boundary conditions are found below.

\section{b. Model domain setup}

The model domain extends $500 \mathrm{~km}$ in the streamwise $(x)$ and spanwise $(y)$ directions, and the model top is at $H=100 \mathrm{~km}$ altitude. The irregularly spaced $x-y-z$ coordinates of the physical domain $\mathcal{D}_{p}$ are mapped onto regularly spaced generalized coordinates $(\bar{x}, \bar{y}, \bar{z})$ of the transformed domain $\mathcal{D}_{t}$ using the bijective transformation (Wedi and Smolarkiewicz 2004; Kühnlein et al. 2012)

$$
(\bar{x}, \bar{y}, \bar{z})=\mathcal{F}(x, y, z): \mathcal{D}_{p} \rightarrow \mathcal{D}_{t} .
$$

Here, the following transformations for the horizontal coordinates have been used (Prusa and Gutowski 2006):

$$
\begin{aligned}
& x(\bar{x})=1+c_{x} \times\left(s_{x 1} \bar{x}+s_{x 2} \bar{x}^{5}\right)-b_{x}, \\
& y(\bar{y})=1+c_{y} \times\left(s_{y 1} \bar{y}+s_{y 2} \bar{y}^{5}\right)-b_{y},
\end{aligned}
$$

where $s_{x 1}=s_{x 2}=s_{y 1}=s_{y 2}=0.5, c_{x}=2 / \gamma_{x}$, and $c_{y}=2 / \gamma_{y}$ by using

$$
\begin{aligned}
& \gamma_{x}=2 s_{x 1}+s_{x 2} \times\left(1-\Delta_{x}\right)^{5}-s_{x 2}\left(-1-\Delta_{x}\right)^{5}, \\
& \gamma_{y}=2 s_{y 1}+s_{y 2} \times\left(1-\Delta_{y}\right)^{5}-s_{y 2}\left(-1-\Delta_{y}\right)^{5}
\end{aligned}
$$

and

$$
\begin{aligned}
& b_{x}=\left[s_{x 1} \times\left(1-\Delta_{x}\right)+s_{x 2} \times\left(1-\Delta_{x}\right)^{5}\right] \times 2 / \gamma_{x} \\
& b_{y}=\left[s_{y 1} \times\left(1-\Delta_{y}\right)+s_{y 2} \times\left(1-\Delta_{y}\right)^{5}\right] \times 2 / \gamma_{y}
\end{aligned}
$$

with $\Delta_{x}=0.015$ and $\Delta_{y}=0.026$ accounting for maximum horizontal resolution at $(x, y)=(-60,0) \mathrm{km}$. The generalized coordinates $(\bar{x}, \bar{y})$ have to be normalized to the interval $[-1,1]$ before applying Eq. (8). The distribution of grid points for simulations 1 and 2 is visualized in Fig. 2 (left panel).

For simulation $1,(n, m, l)=(336,336,501)$ grid points are used in the three coordinate directions resulting in spatial resolutions of $(\Delta x, \Delta y, \Delta z)=(1-6,1-6,0.2) \mathrm{km}$, respectively. For simulation $2,(n, m, l)=(672,672,501)$ grid points are used in the three coordinate directions resulting in spatial resolutions of $(\Delta x, \Delta y, \Delta z)=(0.5-3,0.5-3,0.2)$ $\mathrm{km}$, respectively. The time step $\delta t$ of the numerical integration was $1.5 \mathrm{~s}$.

For the vertical direction we use the nonorthogonal terrainfollowing coordinate

$$
\bar{z}=H \frac{z-z_{s}(x, y)}{H-z_{s}(x, y)},
$$

which assumes an irregular but at least twice differentiable lower boundary $z_{s}(x, y)$. Here, the terrain height $z_{s}$ of Auckland Island is taken from $250 \mathrm{~m}$ resolution ASTER ${ }^{1}$ topography, centered at $(x, y)=(-150,-25) \mathrm{km}$ and sampled on the irregular grid. Auckland Island is a small topographic gravity wave source that is barely resolved in global circulation models. Its maximum terrain elevation reaches $650 \mathrm{~m}$ at $(x, y)=(150,-50) \mathrm{km}$.

The terrain cross-sectional length of $\sim 20-50 \mathrm{~km}$ indicates the potential range of $\lambda_{x}$ for terrain-forced gravity waves. The corresponding intrinsic frequency $\omega_{\mathrm{i}} \approx 2 \pi u_{e}(z) / \lambda_{x}$ profiles for these wavelengths indicate turning levels where $\omega_{i} \approx N$ and the potential for evanescent gravity waves with $\lambda_{x} \leq 20-30 \mathrm{~km}$ in the PNJ, Fig. 2c.

\section{c. Initial and boundary conditions}

The initial and ambient profiles of wind and potential temperature $\left(u_{e}, v_{e}, \theta_{e}\right)$ are taken from the NAVGEM reanalysis (Eckermann et al. 2018). In particular, we use the 0600 UTC profile from 14 July 2014 for the numerical simulations presented in this paper (Eckermann et al. 2016; Broutman et al. 2017). Figure $2 \mathrm{~b}$ shows the respective vertical profiles for $u_{e}$ and $v_{e}$ as well as for the buoyancy frequency $N=\sqrt{g \partial \ln \theta_{e} / \partial z}$. There is southwesterly low-level forcing across Auckland Island; a strong PNJ extending from $z \sim 40$ to $70 \mathrm{~km}$; and a high-negative-shear region above approaching the zero horizontal wind line at $z \approx 82 \mathrm{~km}$, a total critical level for all linear and stationary mountain waves (Teixeira 2014). As mentioned by Broutman et al. (2017), the directional shear is rather weak for this $0600 \mathrm{UTC}$ sounding: the wind direction $\alpha_{H}$ is toward the southeast, with $\alpha_{H}$ between about $-15^{\circ}$ and $-35^{\circ}$. The largest wind rotation rate is $\Delta \alpha_{H} \cong-4.5^{\circ} \mathrm{km}^{-1}$ near $z=66 \mathrm{~km}$.

\footnotetext{
${ }^{1}$ Digital elevation data have been downloaded from https:// asterweb.jpl.nasa.gov/gdem.asp. ASTER Global Digital Elevation Map (GDEM) is a product of Ministry of Economy, Trade, and Industry (METI) and NASA.
} 

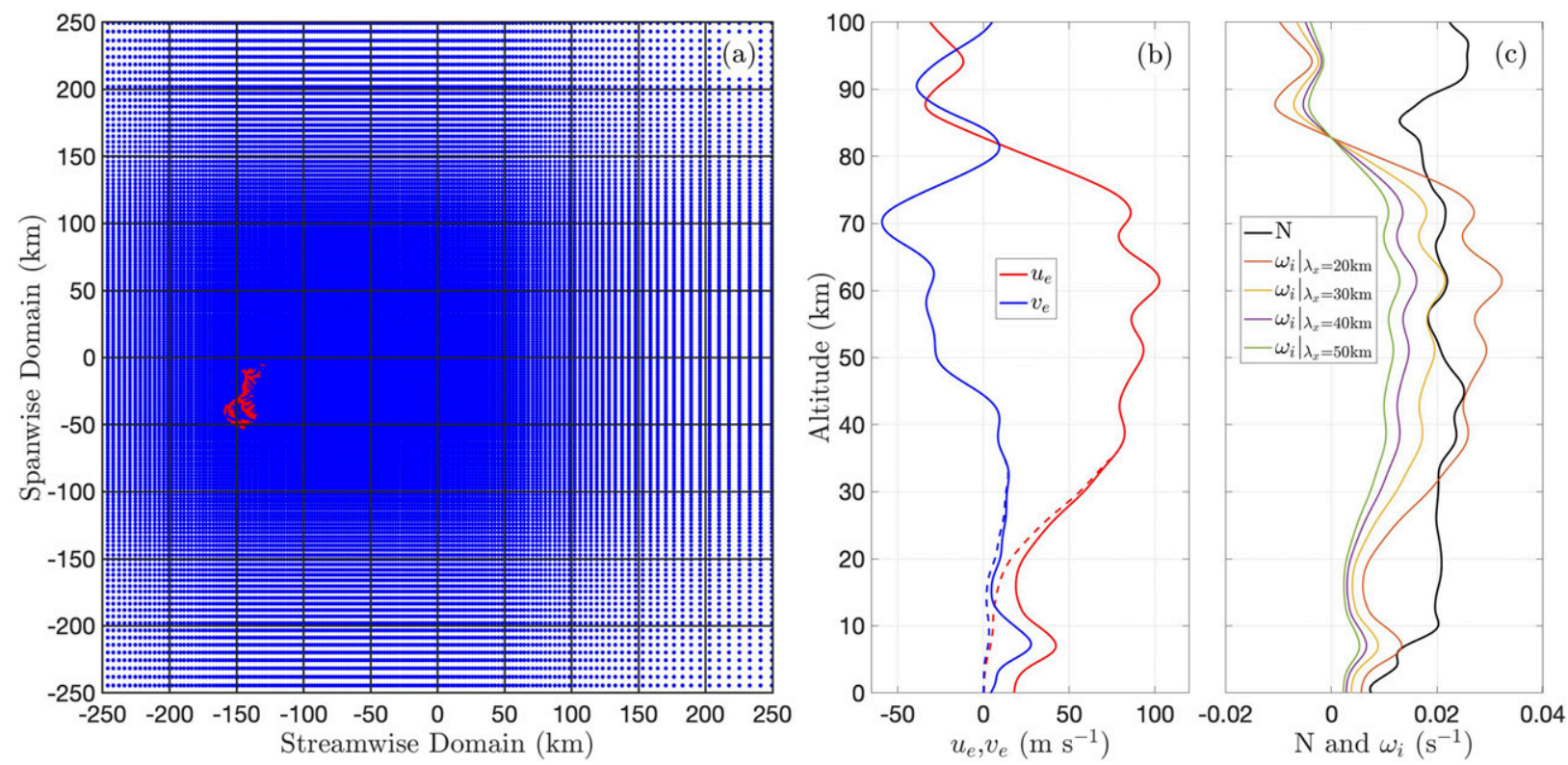

FIG. 2. (a) Horizontal distribution of grid points in the computational domain. The red shaded region marks the outline of Auckland Island. (b) Vertical profiles of the ambient profiles $u_{e}$ and $v_{e}$ (solid lines). The dashed lines mark the respective initial profiles at $t=0$; see text. (c) Brunt-Väisälä frequency $N$ and intrinsic frequency $\omega_{i}$ for selected values $\lambda_{x}$ of stationary mountain waves.

To suppress spurious acoustic modes at high altitudes and the reflection of gravity waves, wave absorbers are used in the horizontal and vertical directions. In the horizontal directions, the absorber time scales $\alpha$ and $\beta$ are implemented as spatial and time-dependent functions. In the vertical direction, we apply a method proposed by Prusa et al. (1996) where the sponge mimics the increasing effect of molecular viscosity with height.

In simulation 2, we additionally apply a gradual ramp up of the horizontal wind in the lowest $30 \mathrm{~km}$ of the model. The respective initial profiles $\left(u_{e 0}, v_{e 0}\right)$ are plotted with dashed lines in Fig. 2b. A series of 2D precursor simulations led to the following sequence minimizing acoustic modes in the mesosphere:

1) simulation of $1200 \mathrm{~s}$ with steady $\left(u_{e 0}, v_{e 0}\right)$ profiles and horizontal sponges temporarily extended to cover the full domain in order to absorb the acoustic gravity wave response from initialization,

2) $1200 \mathrm{~s}$ recession of the horizontal sponges to $60 \mathrm{~km}$ distance from the outer perimeter of the domain, and

3) start of the wind ramp-up at $t_{a}=1800 \mathrm{~s}$ until $t_{b}=7200 \mathrm{~s}$ from the initial to the final ambient wind profiles according to

$$
\begin{aligned}
& u_{e}=u_{e 0}+\left(u_{e}-u_{e 0}\right) \xi(\varsigma) \quad \text { and } \\
& v_{e}=v_{e 0}+\left(v_{e}-v_{e 0}\right) \xi(\varsigma),
\end{aligned}
$$

where $\xi(s) \equiv \min \left[1, \varsigma^{3}\left(1-15 s+6 s^{2}\right)\right]$ and $s=\left(t-t_{a}\right) /\left(t_{b}-t_{a}\right)$. The specific forces $F_{x}$ and $F_{y}$ used in Eq. (1) are the temporal derivatives of Eq. (12).

Because the wind ramp in simulation 2 creates a time delay in the gravity wave evolution, simulation 1 is used for temporal analyses of gravity wave mode propagation times and for comparisons with similar results from Eckermann et al. (2016).

\section{d. Wave analysis}

To allow for gravity wave intrinsic characteristics to vary locally over the horizontal domain, background fields are calculated from the discrete solutions $\Psi_{i}^{n}=\left(\mathbf{u}_{i}^{n}, \theta_{i}^{n}, p_{i}^{n}, T_{i}^{n}\right)$ by averaging over the local streamwise horizontal wavelength $\lambda_{x}$ at all points in space and time. Perturbations associated with gravity waves are then given by

$$
\Psi^{\prime \prime}=\Psi-\langle\Psi\rangle_{\lambda_{x}},
$$

where \langle\rangle indicates streamwise averaging. The quantity $\lambda_{x}$ varies locally and is calculated from $w$ using the horizontal wavelet analysis method of Chen and Chu (2017), a modification of the Torrence and Compo (1998) wavelet analysis method that corrects for amplitude biases in smaller $\lambda_{x}$. The horizontal wavelet analysis returns the spectral amplitudes of each horizontal wavenumber for all discrete points in the domain, from which $\lambda_{x}$ is defined as the spatial scale with the highest spectral power at each point. Horizontal and vertical energy fluxes are determined by

$$
\mathrm{EF}_{x}=\left\langle u^{\prime \prime} p^{\prime \prime}\right\rangle_{\lambda_{x}} \text { and } \mathrm{EF}_{z}=\left\langle w^{\prime \prime} p^{\prime \prime}\right\rangle_{\lambda_{x}},
$$

and the zonal momentum flux is determined by

$$
\mathrm{MF}_{x}=\bar{\rho}\left\langle u^{\prime \prime} w^{\prime \prime}\right\rangle_{\lambda_{x}}
$$

The intrinsic frequency of the mountain waves $\omega_{i}$ in the computational domain is approximated by $\omega_{i}=k\left(c-\langle u\rangle_{\lambda_{x}}\right)$ for streamwise cross sections, using the zonal wavenumber $k=2 \pi / \lambda_{x}$ 

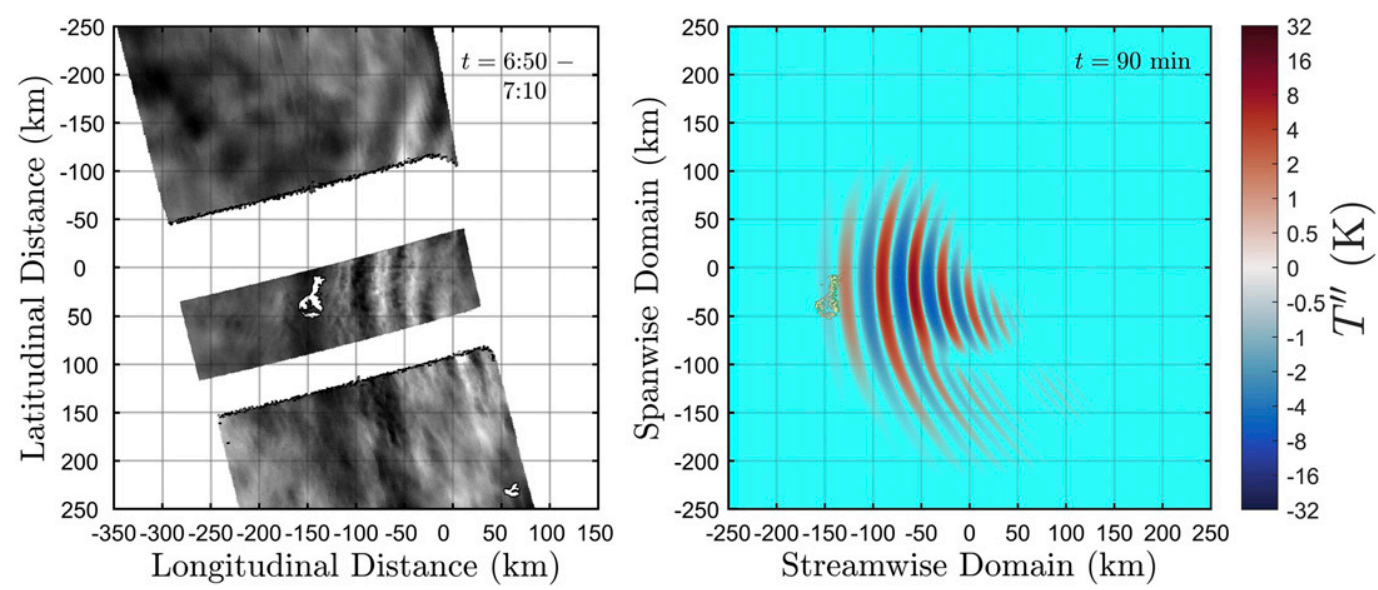

FIG. 3. Overview of simulations and observations of the event. (left) Initial AMTM observation of OH brightness over Auckland Island at $z \approx 83.5 \mathrm{~km}$ [adapted from Pautet et al. (2016) using data provided by P. Dominique Pautet] and (right) simulated temperature perturbations at $z=78 \mathrm{~km}$.

from the horizontal wavelet analysis at all grid points in space and time. The horizontal phase speed $c$ is determined by a wavelet analysis of Hovmöller $z-t$ profiles of vertical velocity $w$ spaced evenly through the domain, giving $k c$ in units of cycles per second. $c$ is then determined using local $\lambda_{x}$ values. The resulting distribution of $\omega_{i}$ values is then compared with the background $N$ as defined above to identify evanescent regions; see section 3 .

\section{Numerical results}

The purpose of the numerical analysis is to demonstrate how tunneling enables small-scale, high-frequency gravity waves to penetrate the PNJ and propagate to the mesopause. We first present the mesospheric gravity wave response in the AMTM from Pautet et al. (2016) and the corresponding characteristics of the simulated gravity wave packet. We then diagnose the stratospheric flow field that enabled deep propagation of the high-frequency orographic gravity wave, showing how tunneling produced additional high-frequency modes that rapidly accumulated amplitude leeward of the hydrostatic modes over the island. The instability evolution reveals a characteristic vorticity morphology driving gravity wave breaking: large streamwise-vertical "mesospheric rolls," which form on alternating gravity wave phases in the shear above the PNJ and descend with time. As the flow becomes more turbulent, apparent horizontal vortex rings reduce the coherence of the streamwise rolls and extend the instability region downward. The resulting momentum deposition produces a deep region of enhanced GWD that extends several hundred kilometers beyond the island.

\section{a. General gravity wave characteristics and observational confirmation}

Figure 3 presents the AMTM observations around 0700 UTC 14 July 2014 and the numerical results of simulation 1 at an elapsed time of $t=90 \mathrm{~min}$. The AMTM observations of Pautet et al. (2016) (left panel) show a sustained gravity wave response over Auckland Island in the retrieved $\mathrm{OH}$ brightness at $z \approx 83.5 \pm 0.5 \mathrm{~km}$ with full width at half maximum (FWHM) $\approx 7.5 \mathrm{~km}$. A progression of up to five wavelengths is visible, with one longer wavelength of weaker brightness intensity directly over the topography and up to four shorter wavelengths with higher brightness intensity extending roughly $200 \mathrm{~km}$ eastward.

Simulated temperature perturbations at $z=78 \mathrm{~km}$ (right panel) capture the dominant gravity wave characteristics observed by the AMTM, with peak $T^{\prime \prime}$ amplitudes of $\pm 10 \mathrm{~K}$ and $\lambda_{x} \approx 40 \mathrm{~km}$ immediately in the lee of the topography and a slightly asymmetric response caused by the southwesterly forcing conditions at the ground. As in Eckermann et al. (2016) and Broutman et al. (2017), the simulation altitude of $z=$ $78 \mathrm{~km}$ is used to indicate peak $T^{\prime \prime}$ amplitudes for the $0600 \mathrm{UTC}$ NAVGEM wind profiles, which occur $\approx 5 \mathrm{~km}$ higher in the AMTM because of the higher critical level in the NAVGEM profiles from 0400 to 0500 UTC (not shown). Successive gravity wave phases extend $\approx 200 \mathrm{~km}$ downwind and exhibit smaller $\lambda_{x}$ and reduced spanwise extents farther downstream. The horizontal geometric spreading and small $\lambda_{x}$ indicate predominantly nonhydrostatic propagation characteristics (Keller 1994) for this extended leeward response (Broutman et al. 2017). The larger $T^{\prime \prime}$ amplitudes of the downwind modes further distinguish these nonhydrostatic modes in both the $\mathrm{OH}$ brightness response and the numerical simulations.

The time evolution of the vertical velocity $(w)$ field in Fig. 4 shows the 3D distribution of the gravity wave as the event evolves, revealing the influence of high-frequency gravity wave modes that quickly reach the MLT and promote instability evolution. The gravity waves extend directly from Auckland Island to $z \approx 80 \mathrm{~km}$, indicating continuous propagation through the PNJ up to the mesopause where they were observed. Cross sections taken at peak amplitude locations (right column) show the center of the gravity wave packet shifting north of the island from $y \approx-25 \mathrm{~km}$ at $t=30 \mathrm{~min}$ to $y \approx 10 \mathrm{~km}$ at later times, suggesting this location for analyzing the gravity wave field in the stratosphere where its orientation aligns with the PNJ. The fastest propagating 

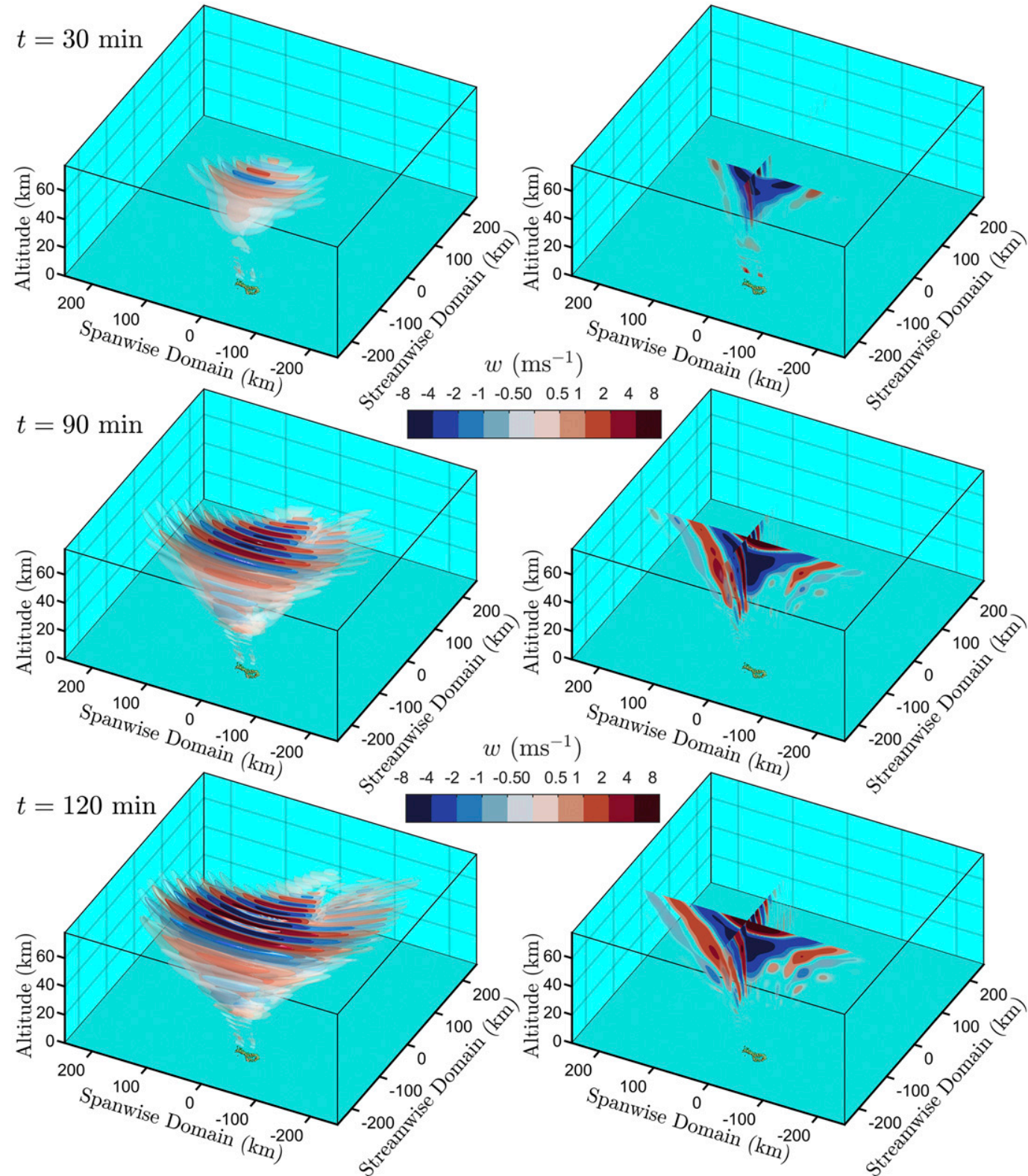

FIG. 4. Time evolution of the simulated 3D vertical velocity ( $w$ ) field shown at elapsed times of (top) 30, (middle) 90 , and (bottom) $120 \mathrm{~min}$. The 3D gravity wave phase structure is presented as both (left) a series of $w$ isosurfaces, plotted on a logarithmic scale with increasing opacity for higher values; and (right) intersecting vertical slices through the domain at $(x, y)=(-130,-25) \mathrm{km}$ for $t=30 \mathrm{~min}$ and $(x, y)=(-90,10) \mathrm{km}$ for $t=90$ and $120 \mathrm{~min}$.

modes reach the MLT in approximately $30 \mathrm{~min}$ (top row), generating an initial response about $50-100 \mathrm{~km}$ east of Auckland Island. After 90 min (middle row), the MLT response matches the characteristics observed in the AMTM, including both 1) the weaker hydrostatic response directly above the topography, which propagates more slowly; and 2) the nonhydrostatic lee waves, which propagate a longer lateral distance to reach the MLT farther downstream. Initial instability structures occur after $120 \mathrm{~min}$ (bottom row), eroding the coherence of two consecutive $w$ phases in the highest amplitude region of the MLT response. These times are notably faster than the $90-240$ min propagation times reported by Broutman et al. (2017), suggesting that these quickly propagating modes may be a tunneling gravity wave response. To diagnose tunneling modes, the next section examines the stratospheric gravity wave characteristics at the packet center and evaluates how the mountain waves propagate through the PNJ.

\section{b. Stratospheric propagation characteristics}

The stratospheric gravity wave characteristics exhibit local amplitude and phase variations throughout the domain, 

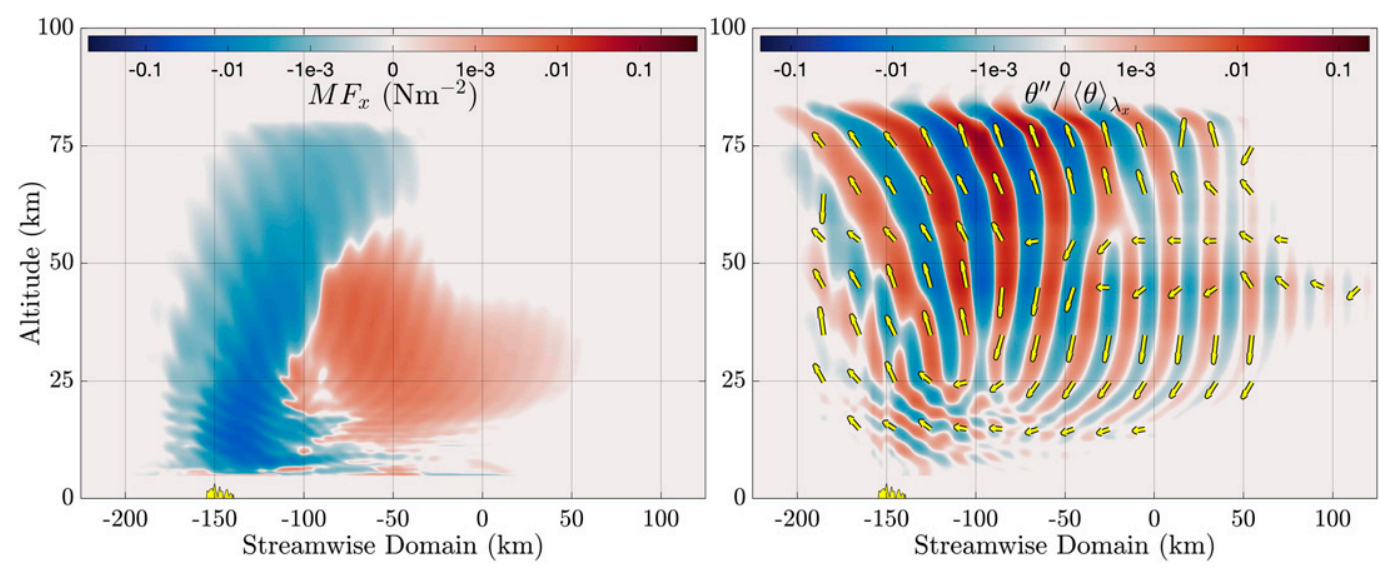

FIG. 5. Streamwise-vertical cross sections of (left) the zonal momentum flux $\mathrm{MF}_{x}$ and (right) the normalized vector quantity EF overlaid on the gravity wave $\theta^{\prime \prime} /\langle\theta\rangle_{\lambda_{x}}$ perturbations at $y=10 \mathrm{~km}$. Negative (positive) $\mathrm{MF}_{x}$ indicates upward (downward) gravity wave propagation. Auckland Island's topography is magnified by a factor of 5 .

indicating where and how gravity wave tunneling enables penetration through the PNJ. Local propagation characteristics are diagnosed through a sequence of metrics: 1) momentum and energy fluxes identify where the gravity wave undergoes vertical propagation, reflection, and ducting; 2) the $\lambda_{x}$ distribution shows how the gravity wave spectra vary in altitude and streamwise distance; and 3) $\omega_{i} / N$ excursions show the locations and magnitudes of evanescent regions. Together, these diagnostics reveal that tunneling gravity wave modes account for the dominant gravity wave response observed above the stratopause.

Figure 5 presents streamwise-vertical cross sections of the momentum and normalized energy fluxes [calculated from Eqs. (14) and (15)] and the associated gravity wave phase structure at $y=10 \mathrm{~km}$. The vector quantity $\mathbf{E F} \equiv\left(\mathrm{EF}_{x}, \mathrm{EF}_{z}\right)$ points perpendicular to the gravity wave phase lines (right panel), which are plotted on a logarithmic color scale to show the full spatial extent of the packet over a broader amplitude range. $\mathrm{MF}_{x}$ indicates where the gravity wave propagates upward (negative $\mathrm{MF}_{x}$ ) and downward (positive $\mathrm{MF}_{x}$ ), while EF shows the direction of gravity wave energy transport at each location in the domain. $\mathrm{EF}_{x}$ points upwind (left) over the entire cross section and marks the full domain as a mountain wave response. $\mathrm{MF}_{x}$ (left panel) indicates the main gravity wave response propagates upward from Auckland Island, exhibiting a large negative $\mathrm{MF}_{x}$ tower (see Kruse and Smith 2015) above and in the lee of the island up to $z \approx 82 \mathrm{~km}$. The negative $\mathrm{MF}_{x}$ tower spreads downstream with altitude as nonhydrostatic modes extend leeward of the stationary hydrostatic response. In this region of upward propagation, the gravity wave tilt is relatively shallow up to $z \approx 25 \mathrm{~km}$, steepening to near-vertical phase lines from $z \sim 30-60 \mathrm{~km}$ and flattening again as the mountain waves approach the total critical level at $z \approx 82 \mathrm{~km}$. Further downstream, positive $\mathrm{MF}_{x}$ indicates net downward propagation of modes below $z \approx 50 \mathrm{~km}$ that are most likely reflected from the PNJ wind maximum at $z \approx 60 \mathrm{~km}$. In this region, the gravity wave phase tilt below $z \approx 50 \mathrm{~km}$ changes from leftward to rightward with a horizontal inflection point near $x \approx-100 \mathrm{~km}$, showing indications of partial and complete reflection in the region below $z \approx 30 \mathrm{~km}$ from $-125 \leq x \leq-100 \mathrm{~km}$ and $-75 \leq$ $x \leq 0 \mathrm{~km}$, respectively.

The area of net-zero $\mathrm{MF}_{x}$ and $\mathrm{EF}_{z}$ within the gravity wave amplitude envelope further identifies a region of compensating upward and downward fluxes where the gravity wave does not exhibit net vertical energy or momentum transport. Leeward of $x \approx-75 \mathrm{~km}$, there is a gap between the dominant positive and negative $\mathrm{MF}_{x}$ regions, which extends downward below the PNJ maximum with downstream distance. In this region, vertical phase lines extend laterally from $-75 \leq x \leq 100 \mathrm{~km}$, and the leftward-pointing $\mathbf{E F}$ arrows indicate a gravity wave response that only transports net energy horizontally. The presence of vertical phase lines where no net vertical energy transport occurs suggests a large evanescent region, which can be diagnosed by examining the gravity wave frequency distribution.

The propagation regime of the mountain waves is diagnosed by Fig. 6, showing the gravity wave $\lambda_{x}$ spectra (left) and the resulting distribution of $\omega_{i} / N$ over the gravity wave packet (right). Streamwise-vertical $\lambda_{x}$ variations determine the local distribution of $\omega_{i}$, showing how intrinsic gravity wave properties vary through the domain. The $\lambda_{x}$ power spectral density (PSD) in Fig. 6 (right) is summed over the streamwise cross section at all altitudes, with vertical profiles of the dominant $\lambda_{x}$ taken from the indicated locations directly in the lee of Auckland Island. The gravity wave response at $x=x_{1}$ has a dominant $\lambda_{x}$ of $\sim 25-30 \mathrm{~km}$ up to $z \approx 25 \mathrm{~km}$. The relatively wide PSD envelope of $\lambda_{x} \sim 20-40 \mathrm{~km}$ and apparent beat pattern show potential reflection of some gravity modes, visible leeward of $x=x_{1}$, but the dominant topographic response in the profiles has $\lambda_{x} \leq 30 \mathrm{~km}$. Above this region, $\lambda_{x}$ increases through the stratospheric jet, reaching maximum values of approximately 30-40 km near $z \sim 70-80 \mathrm{~km}$ where these scales were observed by AMTM and lidar measurements. Stratospheric profiles also show decreasing $\lambda_{x}$ from $x \approx x_{1}$ to 

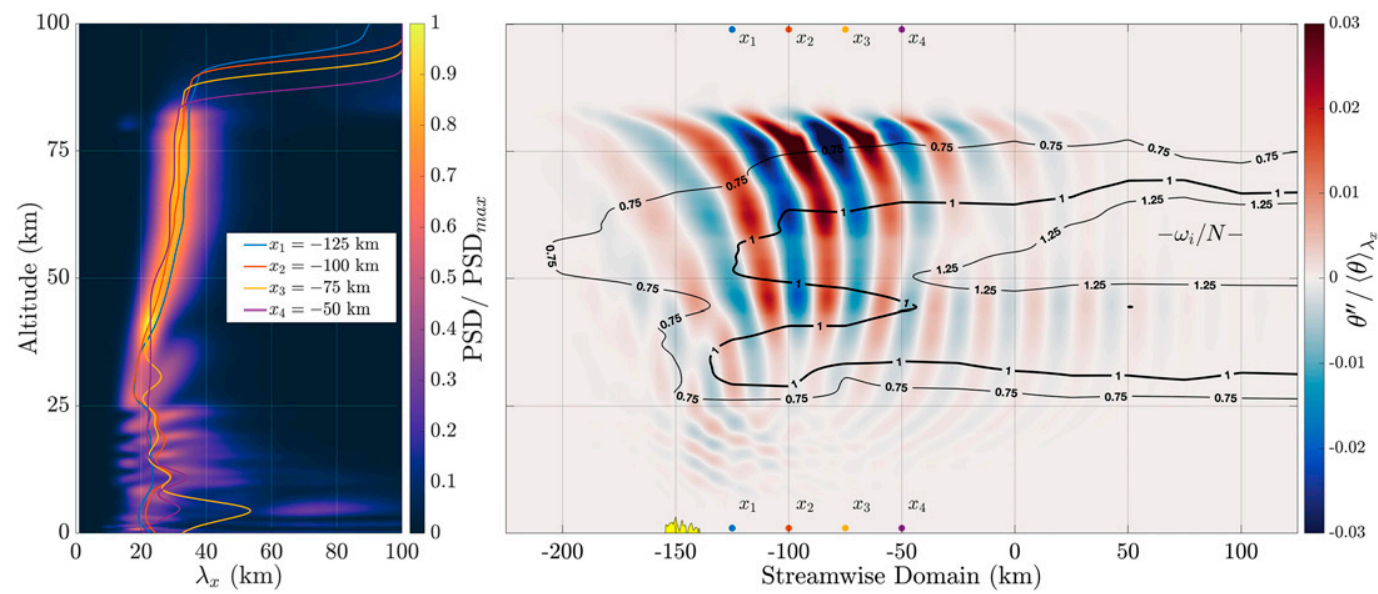

FIG. 6. (left) Gravity wave $\lambda_{x}$ spectra from the wavelet analysis for the whole domain, with profiles drawn at the four locations indicated in the right panel, and (right) streamwise-vertical cross section of $\theta^{\prime \prime} \mid\langle\theta\rangle_{\lambda_{x}}$ with the corresponding $\omega_{i} / N$ contours drawn to indicate evanescent regions. Auckland Island's topography is magnified by a factor of 5 .

$x_{4}$, where nonhydrostatic propagation produces an extended leeward gravity wave response.

The $\omega_{i} / N$ distribution confirms that the highest amplitude gravity wave modes reach MLT by tunneling through the PNJ and explains why the leeward gravity wave response is reflected. Figure 6 (right) displays the same $\theta^{\prime \prime} /\langle\theta\rangle_{\lambda_{x}}$ distribution as in Fig. 5 on a linear scale to emphasize the amplitude decrease in evanescent layers, overlaid by $\omega_{i} / N$ contours that indicate the locations and magnitudes of evanescent regions. Upward propagating mountain waves indicated by $\mathrm{MF}_{x}$ tunnel through two evanescent regions from $z \sim 30-40 \mathrm{~km}$ and $z \sim$ $50-60 \mathrm{~km}$. Gravity wave amplitudes precisely follow $\omega_{i} / N$ contours, with $\theta^{\prime \prime} /\langle\theta\rangle_{\lambda_{x}}$ decreasing in both evanescent regions and increasing again above the PNJ. While the gravity wave modes directly above the topography exhibit larger $\lambda_{x}$ and do not propagate through an evanescent region, the tunneling modes transport more momentum/energy and produce notably higher amplitudes from $x_{1}$ to $x_{4}$ in the MLT. The attenuation in both evanescent levels delays the saturation of these tunneling gravity wave modes to higher altitudes, enabling the mountain waves to penetrate the PNJ and to reach $z \approx 78 \mathrm{~km}$ without becoming convectively unstable. Moving leeward from $x \approx x_{1}$ to $x_{4}$, decreasing $\lambda_{x}$ increases the depth of the top evanescent region, reflecting the gravity wave below $50 \mathrm{~km}$ as seen in the phase tilt and indicated by $\mathrm{MF}_{x}$ and $\mathrm{EF}_{z}$ in Fig. 5. Leeward of $x$ $\approx x_{4}$, the two evanescent regions combine and increase in amplitude, accounting for the nontunneling gravity wave response with no $\mathrm{MF}_{x}$ or $\mathrm{EF}_{z}$ indicated by Fig. 5. The stratospheric propagation characteristics thus reveal $\omega_{i} / N$ as the ultimate arbiter of gravity waves penetrating the PNJ, enabling tunneling modes to produce the dominant high altitude response that leads to rapid instability evolution in the MLT.

\section{c. Evolution of instabilities and gravity wave breaking}

The horizontal fields in Fig. 7 indicate gravity wave breaking leeward of the island as initially 2D, spanwise-uniform instabilities that develop apparent $3 \mathrm{D}$ vortex rings, which expand the breaking region over time. The AMTM OH brightness (left panels) is shown for aircraft overpasses beginning at 1015 (top) and 1105 UTC (bottom) 14 July 2014. The AMTM response is approximated in the manner of Fritts et al. (2014), treating $\theta$ as a passive tracer (Chandran et al. 2012) and applying a Gaussian brightness profile

$$
B(z)=B_{0} \exp \left[-\left(z-z_{\text {layer }}\right)^{2} / 2 \sigma_{z}^{2}\right]
$$

as a vertically weighted average of the simulation data. Here $z_{\text {layer }}$ is the peak brightness altitude, and $\sigma_{z}$ is the standard deviation of the layer, set such that the FWHM depth $z_{\mathrm{FWHM}}=2 \sqrt{2 \ln 2} \sigma_{z}$ corresponds to the desired layer depth. The simulation 2 results are shown at time $t=3 \mathrm{~h} 45$ min and $t=4 \mathrm{~h} 45 \mathrm{~min}$ for the brightness-weighted $\theta$ (center panels) and $w$ (right panels) fields: $\theta$ fields are shown with $\left(z_{\text {layer }}, z_{\mathrm{FWHM}}\right)=(78,7) \mathrm{km}$ to match the vertical sensitivity of the AMTM, while $w$ fields are shown with a narrower $z_{\mathrm{FWHM}}=2 \mathrm{~km}$ and $z_{\text {layer }}=(76,74) \mathrm{km}$ to emphasize shallower vortical structures as they descend.

The top row of Fig. 7 shows the initial 2D instability response forming in the lee of the island. The $\mathrm{OH}$ brightness at 1015 UTC features thin, bright temperature enhancements aligned with and occurring within the brightest (warm) gravity wave phases. The third warm phase east of the island contains two such enhancements in both the $\mathrm{OH}$ brightness and the brightness-weighted $\theta$ field at $t=3 \mathrm{~h} 45 \mathrm{~min}$. The streamwise alignment and periodicity of these features are consistent with Kelvin-Helmholtz instabilities observed from below in airglow and polar mesospheric cloud (PMC) images (see, e.g., Baumgarten and Fritts 2014; Fritts et al. 2017, 2019b), and the brightness-weighted $w$ field further reveals these two successive features to have upward and downward motion indicative of overturning. The adjacent gravity wave $w$ phases exhibit steepened, "sawtooth" amplitude distributions like those observed by Fritts et al. (2019a), which confirm this region as the source of GW breaking. Together these fields suggest a 

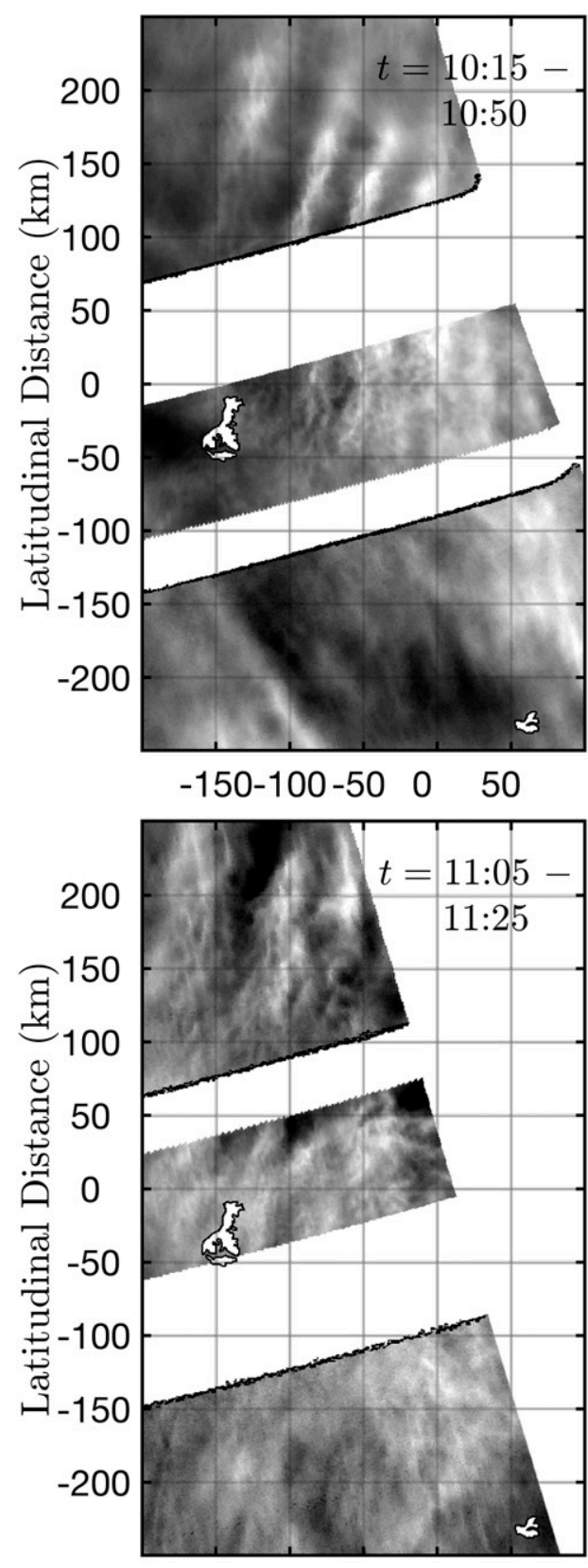

$-150-100-50 \quad 0 \quad 50$

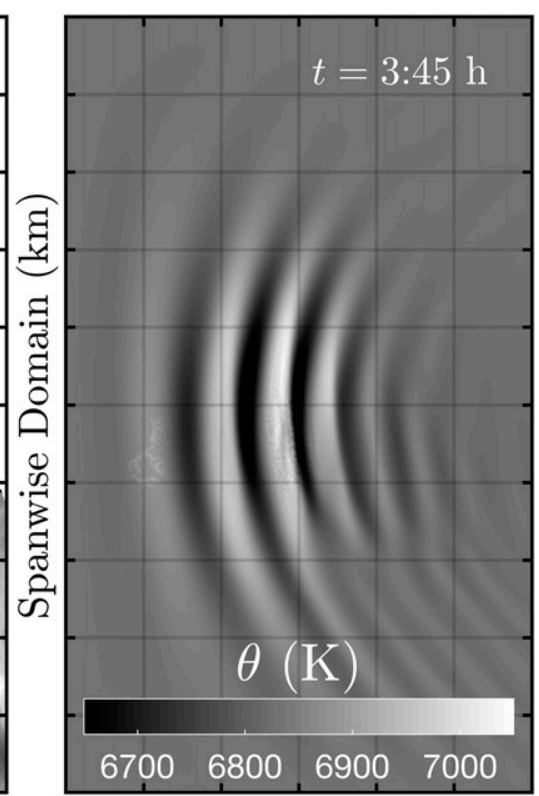

$$
-150-100-50 \quad 0 \quad 50
$$

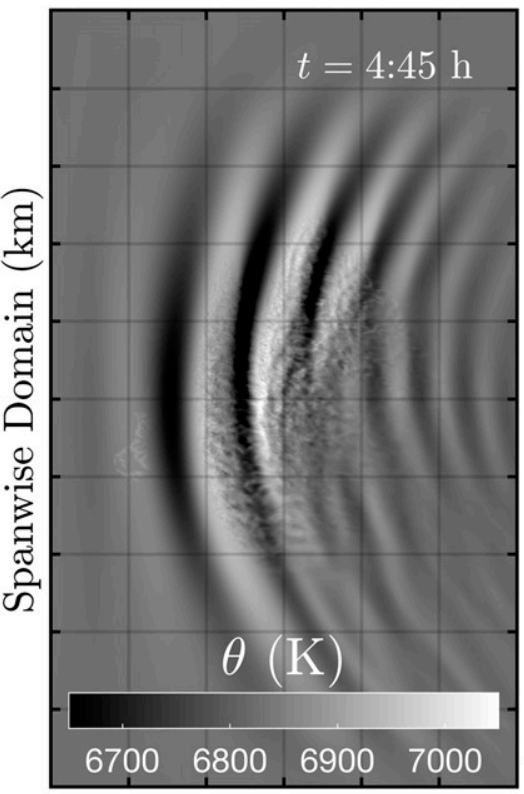

$-150-100-50 \quad 0 \quad 50$

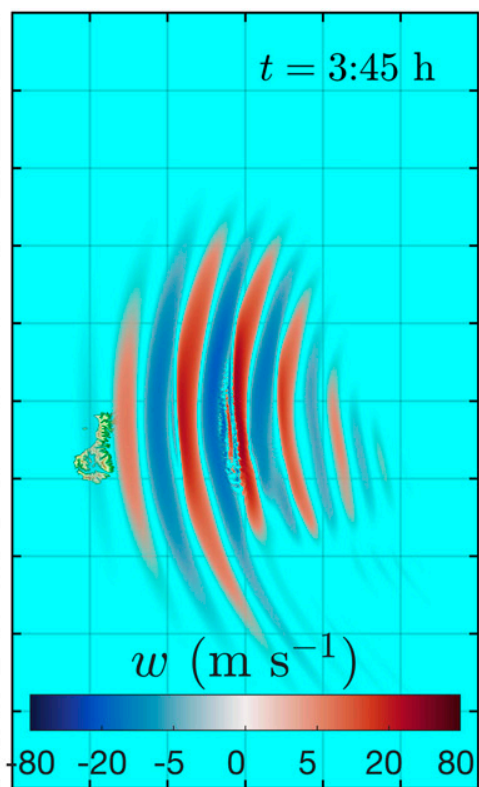

$-150-100-50 \quad 0 \quad 50$

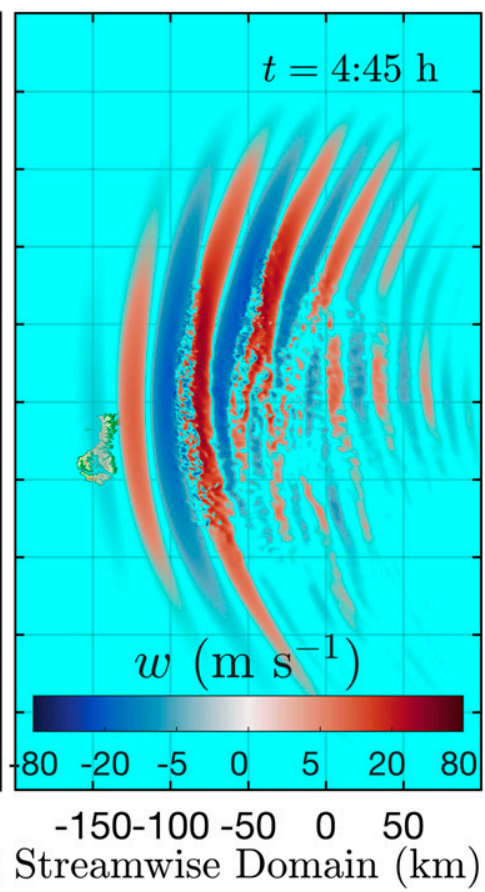

FIG. 7. As in Figs. 3 and 4, but for the instability evolution in the gravity wave $\theta$ and $w$ fields, shown for both (left) the observations [adapted from Pautet et al. (2016) using data provided by P. Dominique Pautet] and the simulation data at (center) the approximate time of the initial instabilities and (right) $1 \mathrm{~h}$ later. The brightness-weighted temperature field is calculated using a $\operatorname{Br}(z) \operatorname{profile}$ with $z_{\mathrm{FWHM}}=$ $7 \mathrm{~km}$ and $z_{\text {layer }}=78 \mathrm{~km}$, while the brightness-weighted $w$ fields have $z_{\mathrm{FWHM}}=2 \mathrm{~km}$ and $z_{\text {layer }}=(76,74) \mathrm{km}$ for $t=3 \mathrm{~h} 45 \mathrm{~min}$ and $4 \mathrm{~h}$ $45 \mathrm{~min}$, respectively.

predominantly $2 \mathrm{D}$, spanwise-uniform initial instability driving local gravity wave breaking, which then expands and develops 3D characteristics over time.

The bottom row of Fig. 7 shows the evolution of these fields after $1 \mathrm{~h}$; all three panels indicate that 3D structures consistent with vortex rings evolve from the initial 2D instability and break up the coherence of the original 2D structures. The $\mathrm{OH}$ brightness at 1105 UTC shows reduced gravity wave coherence and weaker amplitudes where instabilities have dissipated the gravity wave phases. Finescale structures in the AMTM now 

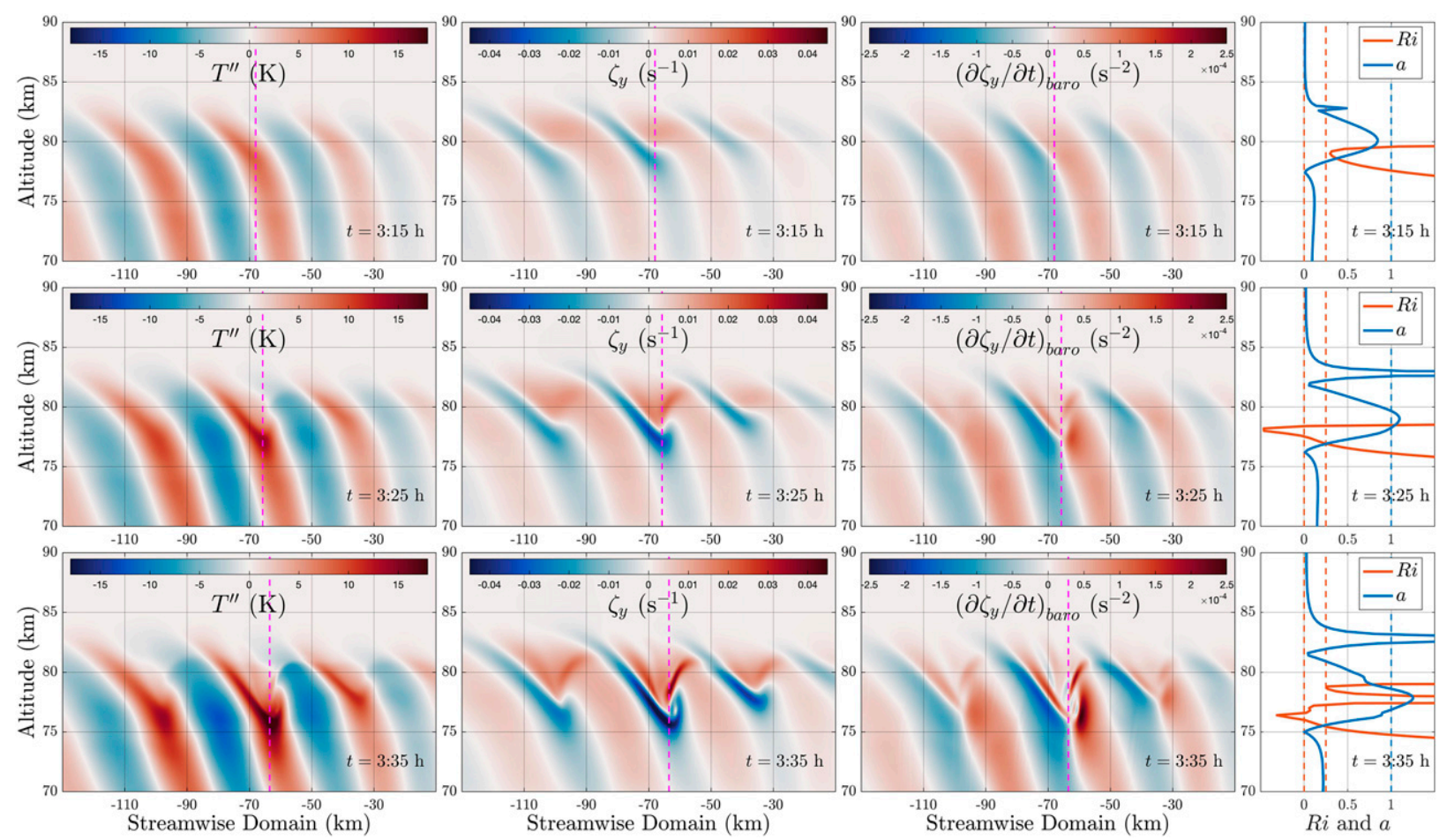

FIG. 8. Streamwise-vertical cross sections of (left) $T^{\prime \prime}$, (left center) $\zeta_{y}$, and (right center) $\left(\partial \zeta_{y} / \partial t\right)_{\text {baro }}$, accompanied by (right) vertical profiles of Ri and $a$ at (top) $t=3 \mathrm{~h} 15 \mathrm{~min}$, (middle) $t=3 \mathrm{~h} 25 \mathrm{~min}$, and (bottom) $t=3 \mathrm{~h} 35 \mathrm{~min}$.

comprise circular rings of enhanced brightness surrounding darker regions that are most pronounced to the northwest of the island. Similar features occur prominently in the brightnessweighted $\theta$ fields stemming from the location of the initial 2D instability at $t=4 \mathrm{~h} 45 \mathrm{~min}$. The brightness-weighted $w$ field suggests that these features denote vortex rings, which commonly arise in convective GW breaking events seen in airglow and PMC images (Miller et al. 2015; Fritts et al. 2017; Hecht et al. 2018; Geach et al. 2020) and idealized simulations (Fritts et al. 2009a,b, 2017, 2021). Rings of upward (red) motion surrounding circles of downward (blue) motion diagnose plunging vortex rings that extend the instability region downward. Northward from the main breaking region, coherent spanwise-uniform features expand laterally along the gravity wave phases as the amplitude increases to promote breaking farther from the center of the packet.

Figure 8 shows how the initial 2D instability forms underlying the horizontal structures, with streamwise-vertical plots at $y=10 \mathrm{~km}$ for $t=3 \mathrm{~h} 15 \mathrm{~min}, 3 \mathrm{~h} 25 \mathrm{~min}$, and $3 \mathrm{~h} 35 \mathrm{~min}$ leading up to the initial simulation time in the horizontal cross sections presented in Fig. 7. $T^{\prime \prime}$ is plotted in the left panels, accompanied by the spanwise vorticity component $\zeta_{y}=\partial u /$ $\partial z-\partial w / \partial x$ in the left-center panels and the baroclinic vorticity source term $\left(\partial \zeta_{y} / \partial t\right)_{\text {baro }}=-\left(g / \theta_{e}\right)\left(\partial \theta^{\prime \prime} / \partial x\right)$ in the right-center panels. At early times, flow motions occur predominantly in the streamwise-vertical plane where $\zeta_{y}$ is the sole vorticity component. Positive (negative) $\zeta_{y}$ indicates clockwise (counterclockwise) streamwise-vertical rotational tendencies. Baroclinic sources and sinks are the dominant $\zeta_{y}$ source term while the flow is primarily $2 \mathrm{D}$ and spanwise stretching/tilting, compressibility effects, and dissipation are weak. Vertical profiles of the convective instability criterion

$$
a=\left|\frac{u^{\prime \prime}}{\langle u\rangle_{\lambda_{x}}}\right|
$$

and the Richardson number

$$
\mathrm{Ri}=\frac{N^{2}}{\left(\frac{\partial u}{\partial z}\right)^{2}}
$$

are plotted in the right panels of Fig. 8 for streamwise locations indicated in the adjacent plots with dashed vertical lines. These profiles identify where the static $(a>1)$ and dynamic $(\mathrm{Ri}<$ 0.25 ) instability criteria are satisfied and provide a qualitative approximation of the initial instability character. These five fields together diagnose how the tunneling gravity waves generate instabilities above the jet that produce the spanwise features seen in the horizontal cross sections.

Adjacent $T^{\prime \prime}$ and $\zeta_{y}$ fields match almost exactly at all times, indicating that baroclinic sources from the horizontal $T^{\prime \prime}$ gradients dominate the initial vorticity evolution. The ambient negative shear above the jet maximum $(z \sim 70-90 \mathrm{~km})$ weakens positive $\zeta_{y}$ phases and amplifies negative $\zeta_{y}$ phases produced by the GW. From $t=3 \mathrm{~h} 15 \mathrm{~min}$ to $3 \mathrm{~h} 25 \mathrm{~min}$, these negative $\zeta_{y}$ sheets thin and intensify along warm $T^{\prime \prime}$ phases, which exhibit enhanced amplitudes where negative $\zeta_{y}$ maxima 

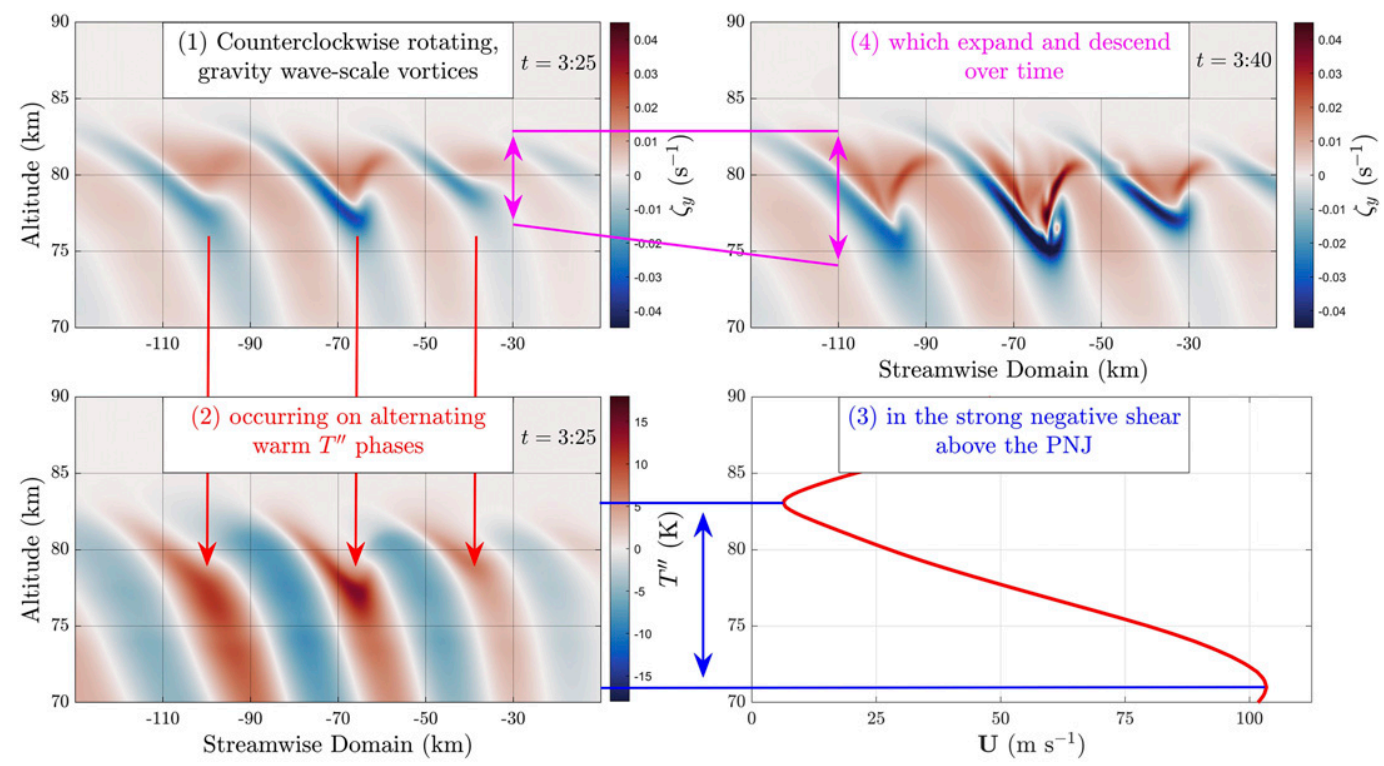

FIG. 9. Schematic of the dominant characteristics of mesospheric rolls. The four defining features are shown in the $\zeta_{y}, T^{\prime \prime}$, and $\mathbf{U}$ fields and identified with text labels.

occur. Pairs of opposite-sign $\left(\partial \zeta_{y} / \partial t\right)_{\text {baro }}$ sheets surround the intensified $\zeta_{y}$ structures at $t=3 \mathrm{~h} 25 \mathrm{~min}$ and $3 \mathrm{~h} 35 \mathrm{~min}$ and drive the development of overturning.

Initial instabilities manifest as counterclockwise rotating vortices on warm gravity wave phases with the strongest negative $\zeta_{y}$. At $t=3 \mathrm{~h} 25 \mathrm{~min}$, critical $\mathrm{Ri}$ is achieved in the warm $T^{\prime \prime}$ phases immediately above $T^{\prime \prime}$ maxima $(z \sim 78-79 \mathrm{~km})$ where $\partial T / \partial z$ becomes negative and $a$ is increasing, satisfying both shear $(0<\mathrm{Ri}<0.25)$ and convective $(\mathrm{Ri}<0) \mathrm{Ri}$ instability criteria. $a=1$ occurs slightly higher at the interface between positive and negative $T^{\prime \prime}(z \sim 79-80 \mathrm{~km})$ coinciding with the local negative $u^{\prime \prime}$ maximum. The central vortex corresponds to the initial spanwise feature seen in the horizontal cross sections at $t=3 \mathrm{~h} 45 \mathrm{~min}$, with additional vortices forming on adjacent warm $T^{\prime \prime}$ phases that follow similar evolutions with short time delays. Primary streamwise-vertical vortices have gargantuan horizontal scales of $\lambda_{x} \approx 40 \mathrm{~km}$, which stem from the local gravity wave $\lambda_{x}$. The instability structure bears remarkable similarity to mountain wave breaking events simulated by Heale et al. (2017) and Fritts et al. (2021), where gravity wave-scale, counterclockwise-rotating vortices form in the negative shear above the Southern Hemisphere PNJ. Heale et al. (2017) also found vortices satisfying both convective and dynamic instability criteria, and analyses by Fritts et al. (2021) confirmed, as we do, that these streamwise instability structures lead to horizontal structures consistent with vortex rings at later times.

We define this unique instability morphology as "mesospheric rolls." Figure 9 shows the four dominant characteristics of this morphology: 1) counterclockwise rotating, gravity wave-scale vortices 2) occurring on alternating warm gravity wave phases 3) in the strong negative shear above the PNJ, 4) which expand and descend over time. The onset of these mesospheric rolls is triggered by the superposition of vorticity sources when a high-amplitude gravity wave propagates through a region of strong negative shear. When a highfrequency gravity wave tunnels through the PNJ, wave attenuation in the evanescent layer delays gravity wave saturation to higher altitudes, thus producing the enhanced gravity wave response needed in the negative shear above the jet maximum. Because the necessary conditions for this instability can be produced anywhere the PNJ occurs, mesospheric rolls can be viewed as a representative instability evolution for this and other high-frequency gravity waves driven to breaking in the negative shear above the PNJ.

Figure 10 shows how the horizontal and vertical instability features expand the breaking region and influence the gravity wave evolution. Streamwise-vertical cross sections (left, $y=$ $10 \mathrm{~km}$ ) and horizontal cross sections (center, $z=78 \mathrm{~km}$ ) of the vorticity magnitude, $|\zeta|=|\nabla \times \mathbf{u}|$, are shown together with 3D $w$ plots (right) at elapsed times of $t=3 \mathrm{~h} 45 \mathrm{~min}$ and $4 \mathrm{~h} 45 \mathrm{~min}$ matching those in Fig. 7. As in Fig. 7, horizontal $|\zeta|$ cross sections employ vertical brightness-weighting with ( $z_{\text {layer }}$, $\left.z_{\text {FWHM }}\right)=(76,2) \mathrm{km}$ to emphasize descending vertical structures. In the streamwise-vertical $|\zeta|$ fields (left), mesospheric rolls descend to lower altitudes as secondary streamwise instabilities engulf the entire breaking region. Spanwise $|\zeta|$ sheets seen in the horizontal panels (center) develop apparent horseshoe vortices and vortex rings that progress to smaller spatial scales and lead to turbulent dissipation. The vertical plunging motion of these structures accompanies the downward motion of the mesospheric rolls and extends the breaking region to a depth of nearly $10 \mathrm{~km}$. Three-dimensional $w$ plots (right) contextualize the vorticity features within the broader evolution of the gravity wave packet. The gravity wave breaking region broadens along positive $w$ phases in the spanwise direction and to additional leeward positive phases in the streamwise direction as amplitudes farther from the instability region 

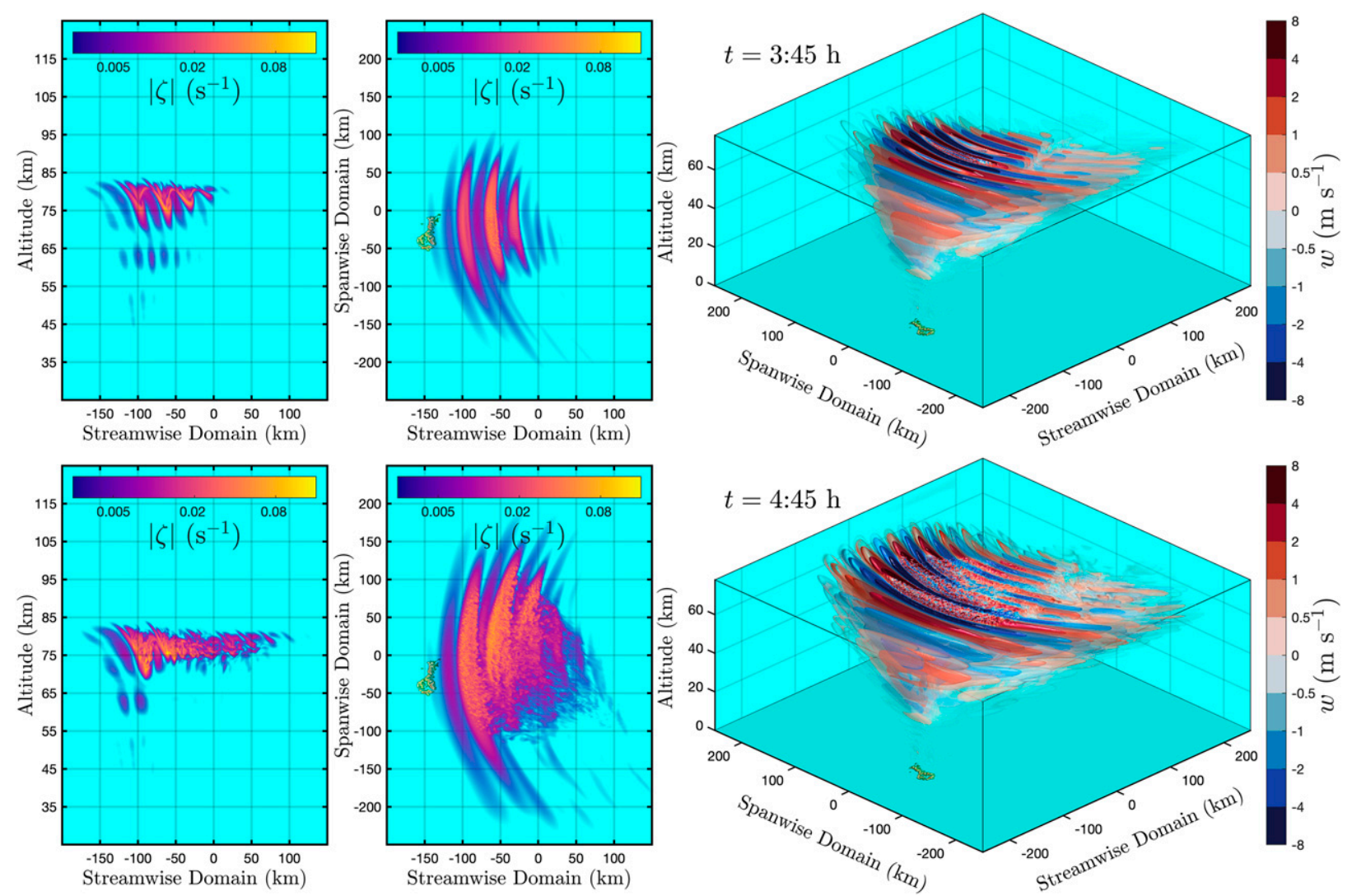

FIG. 10. (left) Streamwise-vertical $(y=10 \mathrm{~km})$ and (center) streamwise-horizontal $\left(z_{\text {layer }}=76 \mathrm{~km}, z_{\mathrm{FWHM}}=2 \mathrm{~km}\right) \mathrm{cross}$ sections of the vorticity magnitude $|\zeta|$ and (right) $3 \mathrm{D} w$ fields shown at elapsed times of $t=3 \mathrm{~h} 45 \mathrm{~min}$ and $4 \mathrm{~h} 45 \mathrm{~min}$ matching those in Fig. 7.

become convectively unstable. Within $1 \mathrm{~h}$ of the initial instability formation, the breaking region extends horizontally over $\mathrm{a} \approx 200 \times 200 \mathrm{~km}^{2}$ region that encompasses nearly the full volume of the initial gravity wave packet from Fig. 3 . The large extent of the breaking region together with the high amplitudes of the gravity wave packet yield high momentum fluxes and a broad region of enhanced GWD above the PNJ.

\section{d. Momentum deposition}

The peak momentum flux per unit mass $\left\langle u^{\prime \prime} w^{\prime \prime}\right\rangle_{\lambda_{x}}$ and GWD $=-\partial\left\langle u^{\prime \prime} w^{\prime \prime}\right\rangle_{\lambda_{x}} / \partial z$ resulting from this event produce an expansive region of decelerated flow above the PNJ. Figure 11 shows the peak $\left\langle u^{\prime \prime} w^{\prime \prime}\right\rangle_{\lambda_{x}}$ and GWD profiles time-averaged over the instability evolution, with shaded regions indicating the standard deviation and the predicted profiles from Eckermann et al. (2016) shown in black. GWD occurs at a lower altitude and over a much larger vertical extent than estimated by Eckermann et al. (2016) using linear saturation theory, with peak deceleration altitudes corresponding to the descended streamwise vortices seen in Fig. 8. The time-averaged profiles indicate sustained $\left\langle u^{\prime \prime} w^{\prime \prime}\right\rangle_{\lambda_{x}}$ in excess of $-400 \mathrm{~m}^{2} \mathrm{~s}^{2}$ and GWD greater than $-800 \mathrm{~m} \mathrm{~s}^{-1} \mathrm{~h}^{-1}$ during gravity wave breaking. These extreme amplitudes are comparable to the MLT gravity wave breaking event documented by Fritts et al. (2018) for a
DEEPWAVE research flight over the Southern Island of New Zealand. There, they estimated sustained momentum fluxes of $-600 \mathrm{~m}^{2} \mathrm{~s}^{-2}$ as "among the largest sustained momentum fluxes observed in the mesosphere."

\section{Summary and conclusions}

Three-dimensional nonlinear numerical simulations were conducted to investigate the vertical and horizontal propagation of internal gravity waves excited by the flow across Auckland Island up to the mesosphere. The numerical simulations reproduced the observed wave pattern in the $\mathrm{OH}$ airglow layer and attained corresponding horizontal wavelengths and temperature amplitudes. The inclusion of tunneling modes and full instability characterization augment previous findings for this event with several important takeaways, emphasizing that tunneling of nonhydrostatic mountain waves through an evanescent layer at the PNJ is the dominant process that enables quick penetration into the MLT. Crucially, tunneling gravity wave modes accelerate amplitude accrual above the PNJ and generate larger instabilities and a deeper gravity wave breaking region than estimated by Eckermann et al. (2016) and Pautet et al. (2016).

The three-dimensional numerical simulations revealed huge overturning vortices in the negative shear above the PNJ that 


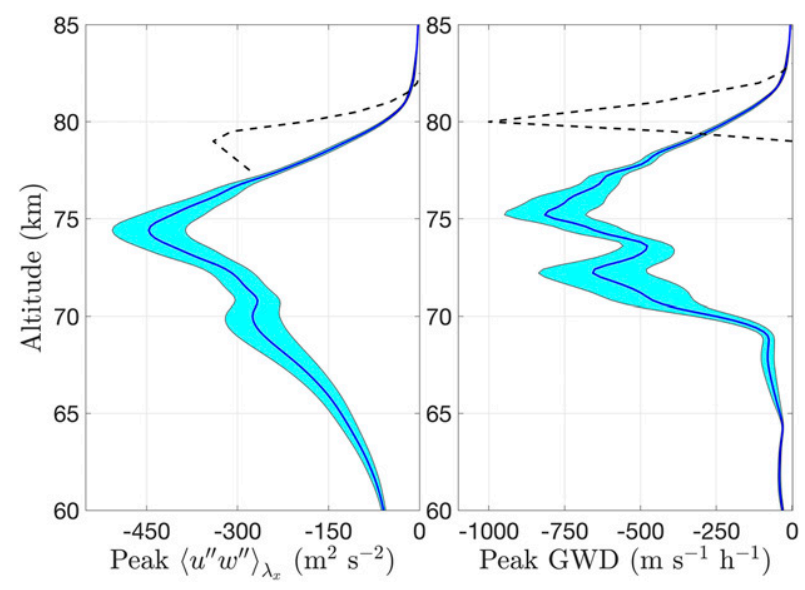

FIG. 11. Time-averaged peak (left) momentum flux per unit mass $\left\langle u^{\prime \prime} w^{\prime \prime}\right\rangle_{\lambda_{x}}$ and (right) GWD after the initial instability onset. Peak GWD occurs at a lower altitude than the theoretical level estimated by Eckermann et al. (2016) from linear saturation theory (shown in dashed black lines) because the vortical structures descend and expand over time.

propagated downward along the warm phases of the mountain waves. The vortical evolution of these "mesospheric rolls" represents a new class of instability morphology, following a characteristic sequence of events that defines the expected behavior for high-frequency gravity waves breaking above the PNJ. The resulting momentum deposition and GWD attain huge values comparable to the values reported by Fritts et al. (2019a) and Taylor et al. (2019) and agree quantitatively with the estimates by Pautet et al. (2016) and Eckermann et al. (2006a). Because the Auckland Island case produces such large, sustained momentum fluxes from a "roughness mode". scale topography source, there is a high likelihood that other small-scale sources in the Southern Hemisphere could excite a similarly large response in the MLT.

Gravity wave tunneling enables small-scale tropospheric sources to have a significant impact on the MLT, contributing to faster gravity wave propagation that leads to amplitude accrual above the PNJ and rapid instability evolution, which produces large regions of GWD. The long duration and extended latitude coverage of the PNJ suggest broad applicability of the propagation dynamics in this study to subantarctic islands and other small-scale, midlatitude gravity wave sources in the Southern Hemisphere. These subgrid-scale gravity wave modes have the potential to produce significant accumulated GWD in the Southern Hemisphere winter MLT. The continued absence of this MLT GWD source in global and mesoscale climate modeling suggests that addressing gravity wave tunneling dynamics is a viable approach to improve the accuracy of climate modeling in the Southern Hemisphere.

Acknowledgments. This research was conducted within the scope of the German research initiative Role of the Middle Atmosphere in Climate (ROMIC) under Grant 01LG1206A by the German Ministry for Education and Research. Funding was also provided by the German Science Foundation (DFG) via the research unit MS-GWaves (GW-TP/DO 1020/9-1 and PACOG/RA 1400/6-1). The AMTM figure data were provided by P. Dominique Pautet. Wavelet analysis routines were provided by C. Chen. Figures 3-7 and 9 employ the cmocean balance color map generated by Thyng et al. (2016).

Data availability statement. The individual 3D datasets used in this study are too large to provide online. The 2D fields used to generate the figures in this study are available for download at https://doi.org/10.6084/m9.figshare.14390510.

\section{REFERENCES}

Alexander, M. J., and A. W. Grimsdell, 2013: Seasonal cycle of orographic gravity wave occurrence above small islands in the Southern Hemisphere: Implications for effects on the general circulation. J. Geophys. Res. Atmos., 118, 11589-11599, https://doi.org/10.1002/2013JD020526.

, S. D. Eckermann, D. Broutman, and J. Ma, 2009: Momentum flux estimates for South Georgia Island mountain waves in the stratosphere observed via satellite. Geophys. Res. Lett., 36, L12816, https://doi.org/10.1029/2009GL038587.

Baumgarten, G., and D. C. Fritts, 2014: Quantifying Kelvin-Helmholtz instability dynamics observed in noctilucent clouds: 1 . Methods and observations. J. Geophys. Res. Atmos., 119, 9324-9337, https://doi.org/10.1002/2014JD021832.

Bramberger, M., and Coauthors, 2017: Does strong tropospheric forcing cause large-amplitude mesospheric gravity waves? A DEEPWAVE case study. J. Geophys. Res. Atmos., 122, 11 422-11 443, https://doi.org/10.1002/2017JD027371.

Broutman, D., S. D. Eckermann, and J. W. Rottman, 2009: Practical application of two-turning-point theory to mountainwave transmission through a wind jet. J. Atmos. Sci., 66, 481494, https://doi.org/10.1175/2008JAS2786.1.

- _ _ - H. Knight, and J. Ma, 2017: A stationary phase solution for mountain waves with application to mesospheric mountain waves generated by Auckland Island. J. Geophys. Res. Atmos., 122, 699-711, https://doi.org/10.1002/2016JD025699.

Chandran, A., D. W. Rusch, G. E. Thomas, S. E. Palo, G. Baumgarten, E. J. Jensen, and A. W. Merkel, 2012: Atmospheric gravity wave effects on polar mesospheric clouds: A comparison of numerical simulations from CARMA 2D with AIM observations. J. Geophys. Res., 117, D20104, https://doi.org/10.1029/ 2012JD017794.

Chen, C., and X. Chu, 2017: Two-dimensional Morlet wavelet transform and its application to wave recognition methodology of automatically extracting two-dimensional wave packets from lidar observations in Antarctica. J. Atmos. Sol.-Terr. Phys., 162, 28-47, https://doi.org/10.1016/j.jastp.2016.10.016.

Clark, T. L., 1977: A small-scale dynamic model using a terrainfollowing coordinate transformation. J. Comput. Phys., 24, 186-215, https://doi.org/10.1016/0021-9991(77)90057-2.

Drob, D. P., and Coauthors, 2015: An update to the Horizontal Wind Model (HWM): The quiet time thermosphere. Earth Space Sci., 2, 301-319, https://doi.org/10.1002/2014EA000089.

Eckermann, S. D., D. Broutman, J. Ma, and J. Lindeman, 2006a: Fourier-ray modeling of short-wavelength trapped lee waves observed in infrared satellite imagery near Jan Mayen. Mon. Wea. Rev., 134, 2830-2848, https://doi.org/ 10.1175/MWR3218.1.

, A. Dörnbrack, H. Flentje, S. B. Vosper, M. J. Mahoney, T. P. Bui, and K. S. Carslaw, 2006b: Mountain wave-induced polar stratospheric cloud forecasts for aircraft science flights during 
SOLVE/THESEO 2000. Wea. Forecasting, 21, 42-68, https:// doi.org/10.1175/WAF901.1.

— , and Coauthors, 2016: Dynamics of orographic gravity waves observed in the mesosphere over the Auckland Islands during the Deep Propagating Gravity Wave Experiment (DEEPWAVE). J. Atmos. Sci., 73, 3855-3876, https://doi.org/10.1175/JAS-D-160059.1 .

— mospheric reanalysis system: Description and application to the 2014 austral winter of the Deep Propagating Gravity Wave Experiment (DEEPWAVE). Mon. Wea. Rev., 146, 26392666, https://doi.org/10.1175/MWR-D-17-0386.1.

Fritts, D. C., L. Wang, J. A. Werne, T. S. Lund, and K. Wan, 2009a: Gravity wave instability dynamics at high Reynolds numbers. Part I: Wave field evolution at large amplitudes and high frequencies. J. Atmos. Sci., 66, 1126-1148, https://doi.org/ 10.1175/2008JAS2727.1.

$-,-,-\longrightarrow,-$, and,$- 2009 \mathrm{~b}$ : Gravity wave instability dynamics at high Reynolds numbers. Part II: Turbulence evolution, structure, and anisotropy. J. Atmos. Sci., 66, 11491171, https://doi.org/10.1175/2008JAS2727.1.

—, K. Wan, J. A. Werne, T. S. Lund, and J. H. Hecht, 2014: Modeling the implications of Kelvin-Helmholtz instability dynamics for airglow observations. J. Geophys. Res. Atmos., 119, 8858-8871, https://doi.org/10.1002/2014JD021737.

— Experiment (DEEPWAVE): An airborne and ground-based exploration of gravity wave propagation and effects from their sources throughout the lower and middle atmosphere. Bull. Amer. Meteor. Soc., 97, 425-453, https://doi.org/10.1175/ BAMS-D-14-00269.1.

— , and Coauthors, 2017: High-resolution observations and modeling of turbulence sources, structures, and intensities in the upper mesosphere. J. Atmos. Sol. Terr. Phys., 162, 57-78, https://doi.org/10.1016/J.JASTP.2016.11.006.

_- and Coauthors, 2018: Large-amplitude mountain waves in the mesosphere accompanying weak cross-mountain flow during DEEPWAVE research flight RF22. J. Geophys. Res. Atmos., 123, 9992-10 022, https://doi.org/10.1029/2017JD028250.

— , L. Wang, M. J. Taylor, P.-D. Pautet, N. R. Criddle, B. Kaifler, S. D. Eckermann, and B. Liley, 2019a: Large-amplitude mountain waves in the mesosphere observed on 21 June 2014 during DEEPWAVE: 2. Nonlinear dynamics, wave breaking, and instabilities. J. Geophys. Res. Atmos., 124, $10006-10032$, https://doi.org/10.1029/2019JD030899.

— and instability dynamics in the summer mesosphere using polar mesospheric cloud imaging and profiling from a stratospheric balloon. J. Geophys. Res. Atmos., 124, 6423-6443, https://doi.org/10.1029/2019JD030298.

_ - T. S. Lund, K. Wan, and H.-L. Liu, 2021: Numerical simulation of mountain waves over the southern Andes. Part II: Momentum fluxes and wave/mean-flow interactions. $J$. Atmos. Sci., https://doi.org/10.1175/JAS-D-20-0207.1, in press.

Gal-Chen, T., and R. C. Somerville, 1975: On the use of a coordinate transformation for the solution of the Navier-Stokes equations. J. Comput. Phys., 17, 209-228, https://doi.org/ 10.1016/0021-9991(75)90037-6.

Geach, C., and Coauthors, 2020: Gravity wave breaking and vortex ring formation observed by PMC Turbo. J. Geophys. Res. Atmos., 125, e2020JD033038, https://doi.org/10.1029/ 2020JD033038.
Gjevik, B., and T. Marthinsen, 1978: Three-dimensional lee-wave pattern. Quart. J. Roy. Meteor. Soc., 104, 947-957, https:// doi.org/10.1002/qj.49710444207.

Heale, C. J., K. Bossert, J. B. Snively, D. C. Fritts, P. Pautet, and M. J. Taylor, 2017: Numerical modeling of a multiscale gravity wave event and its airglow signatures over Mount Cook, New Zealand, during the DEEPWAVE campaign. J. Geophys. Res. Atmos., 122, 846-860, https://doi.org/10.1002/2016JD025700.

Hecht, J. H., and Coauthors, 2018: Observations of the breakdown of mountain waves over the Andes Lidar Observatory at Cerro Pachon on 8/9 July 2012. J. Geophys. Res. Atmos., 123, 276-299, https://doi.org/10.1002/2017JD027303.

Hendricks, E. A., J. D. Doyle, S. D. Eckermann, Q. Jiang, and P. A. Reinecke, 2014: What is the source of the stratospheric gravity wave belt in austral winter? J. Atmos. Sci., 71, 1583-1592, https://doi.org/10.1175/JAS-D-13-0332.1.

Hindley, N. P., C. J. Wright, N. D. Smith, L. Hoffmann, L. A. Holt, M. J. Alexander, T. Moffat-Griffin, and N. J. Mitchell, 2019: Gravity waves in the winter stratosphere over the Southern Ocean: High-resolution satellite observations and 3-D spectral analysis. Atmos. Chem. Phys., 19, 15377-15414, https://doi.org/ 10.5194/acp-19-15377-2019.

Hoffmann, L., A. W. Grimsdell, M. J. Alexander, and A. Dörnbrack, 2016: Stratospheric gravity waves at Southern Hemisphere orographic hotspots: 2003-2014 AIRS/Aqua observations. Atmos. Chem. Phys., 16, 9381-9397, https://doi.org/10.5194/acp-16-93812016.

Hogan, T. F., and Coauthors, 2014: The Navy Global Environmental Model. Oceanography, 27 (3), 116-125, https://doi.org/10.5670/ oceanog.2014.73.

Keller, T. L., 1994: Implications of the hydrostatic assumption on atmospheric gravity waves. J. Atmos. Sci., 51, 1915-1929, https://doi.org/10.1175/1520-0469(1994)051<1915:IOTHAO> 2.0.CO;2.

Kruse, C. G., and R. B. Smith, 2015: Gravity wave diagnostics and characteristics in mesoscale fields. J. Atmos. Sci., 72, 43724392, https://doi.org/10.1175/JAS-D-15-0079.1.

,-- , and S. D. Eckermann, 2016: The midlatitude lowerstratospheric mountain wave "valve layer." J. Atmos. Sci., 73, 5081-5100, https://doi.org/10.1175/JAS-D-16-0173.1.

Kühnlein, C., P. K. Smolarkiewicz, and A. Dörnbrack, 2012: Modelling atmospheric flows with adaptive moving meshes. J. Comput. Phys., 231, 2741-2763, https://doi.org/10.1016/j.jcp.2011.12.012.

Li, J., T. Li, Q. Wu, Y. Tang, Z. Wu, and J. Cui, 2020: Characteristics of small-scale gravity waves in the Arctic winter mesosphere. J. Geophys. Res. Space Phys., 125, e2019JA027643, https:// doi.org/10.1029/2019JA027643.

Lund, T. S., D. C. Fritts, K. Wan, B. Laughman, and H. L. Liu, 2020: Numerical simulation of mountain waves over the southern Andes. Part I: Mountain wave and secondary wave character, evolutions, and breaking. J. Atmos. Sci., 77, 4337-4356, https:// doi.org/10.1175/JAS-D-19-0356.1.

Miller, A., and Coauthors, 2015: Stratospheric imaging of polar mesospheric clouds: A new window on small-scale atmospheric dynamics. Geophys. Res. Lett., 42, 6058-6065, https:// doi.org/10.1002/2015GL064758.

Mitchell, R. M., R. P. Cechet, P. J. Turner, and C. C. Elsum, 1990: Observation and interpretation of wave clouds over Macquarie Island. Quart. J. Roy. Meteor. Soc., 116, 741-752, https://doi.org/ 10.1002/qj.49711649310.

Nault, J. T., and B. R. Sutherland, 2008: Beyond ray tracing for internal waves. I. small-amplitude anelastic waves. Phys. Fluids, 20, 106601, https://doi.org/10.1063/1.2993167. 
Pautet, P.-D., and Coauthors, 2016: Large-amplitude mesospheric response to an orographic wave generated over the Southern Ocean Auckland Islands (50.7 S). J. Geophys. Res. Atmos., 121, 1431-1441, https://doi.org/10.1002/2015JD024336.

—, M. J. Taylor, S. D. Eckermann, and N. Criddle, 2019: Regional distribution of mesospheric small-scale gravity waves during DEEPWAVE. J. Geophys. Res. Atmos., 124, 7069-7081, https://doi.org/10.1029/2019JD030271.

Picone, J., A. Hedin, D. P. Drob, and A. Aikin, 2002: NRLMSISE00 empirical model of the atmosphere: Statistical comparisons and scientific issues. J. Geophys. Res. Space Phys., 107, 1468, https://doi.org/10.1029/2002JA009430.

Plougonven, R., A. Hertzog, and M. J. Alexander, 2015: Case studies of nonorographic gravity waves over the Southern Ocean emphasize the role of moisture. J. Geophys. Res. Atmos., 120, 12781299, https://doi.org/10.1002/2014JD022332.

— , V. Jewtoukoff, A. de la Cámara, F. Lott, and A. Hertzog, 2017: On the relation between gravity waves and wind speed in the lower stratosphere over the Southern Ocean. J. Atmos. Sci., 74, 1075-1093, https://doi.org/10.1175/JAS-D-16-0096.1.

Prusa, J. M., and P. K. Smolarkiewicz, 2003: An all-scale anelastic model for geophysical flows: Dynamic grid deformation. J. Comput. Phys., 190, 601-622, https://doi.org/10.1016/S00219991(03)00299-7.

—_, and W. J. Gutowski, 2006: MPDATA and grid adaptivity in geophysical fluid flow models. Int. J. Numer. Methods Fluids, 50, 1207-1228, https://doi.org/10.1002/fld.1152.

_ breaking at high altitudes of gravity waves excited by tropospheric forcing. J. Atmos. Sci., 53, 2186-2216, https://doi.org/ 10.1175/1520-0469(1996)053<2186:PABAHA > 2.0.CO;2.

,-- , and A. A. Wyszogrodzki, 2008: EULAG, a computational model for multiscale flows. Comput. Fluids, 37, 1193 1207, https://doi.org/10.1016/j.compfluid.2007.12.001.

Schoeberl, M. R., 1985: The penetration of mountain waves into the middle atmosphere. J. Atmos. Sci., 42, 2856-2864, https://doi.org/ 10.1175/1520-0469(1985)042<2856:TPOMWI>2.0.CO;2.

Sharman, R. D., and M. G. Wurtele, 1983: Ship waves and lee waves. J. Atmos. Sci., 40, 396-427, https://doi.org/10.1175/ 1520-0469(1983)040<0396:SWALW >2.0.CO;2.

$\longrightarrow$, and — 2004: Three-dimensional structure of forced gravity waves and lee waves. J. Atmos. Sci., 61, 664-681, https://doi.org/ 10.1175/1520-0469(2004)061<0664:TSOFGW>2.0.CO;2.

Smith, R. B., 1989: Hydrostatic airflow over mountains. $A d v$. Geophys., 31, 1-41, https://doi.org/10.1016/S0065-2687(08)60052-7.

— J. Atmos. Sci., 74, 1381-1402, https://doi.org/10.1175/JAS-D16-0297.1.

Smolarkiewicz, P. K., and L. G. Margolin, 1997: On forward-in-time differencing for fluids: an Eulerian/semi-Lagrangian non-hydrostatic model for stratified flows. Atmos.-Ocean, 35, 127-152, https:// doi.org/10.1080/07055900.1997.9687345.

_ , and - 1998: MPDATA: A finite-difference solver for geophysical flows. J. Comput. Phys., 140, 459-480, https:// doi.org/10.1006/jcph.1998.5901.

— for multiscale flows: An MHD extension. J. Comput. Phys., 236, 608-623, https://doi.org/10.1016/j.jcp.2012.11.008.

_ C. Kühnlein, and N. P. Wedi, 2014: A consistent framework for discrete integrations of soundproof and compressible PDEs of atmospheric dynamics. J. Comput. Phys., 263, 185205, https://doi.org/10.1016/j.jcp.2014.01.031.

,-- , and,- 2019 : Semi-implicit integrations of perturbation equations for all-scale atmospheric dynamics. J. Comput. Phys., 376, 145-159, https://doi.org/10.1016/j.jcp.2018.09.032.

Sutherland, B. R., and K. Yewchuck, 2004: Internal wave tunnelling. J. Fluid Mech., 511, 125-134, https://doi.org/10.1017/ S0022112004009863.

Taylor, M. J., and Coauthors, 2019: Large-amplitude mountain waves in the mesosphere observed on 21 June 2014 during DEEPWAVE: 1. Wave development, scales, momentum fluxes, and environmental sensitivity. J. Geophys. Res. Atmos., 124, 10 364-10 384, https://doi.org/10.1029/2019JD030932.

Teixeira, M. A. C., 2014: The physics of orographic gravity wave drag. Front. Phys., 2, 43, https://doi.org/10.3389/ fphy.2014.00043.

Thyng, K. M., C. A. Greene, R. D. Hetland, H. M. Zimmerle, and S. F. DiMarco, 2016: True colors of oceanography: Guidelines for effective and accurate colormap selection. Oceanography, 29 (3), 9-13, https://doi.org/10.5670/oceanog.2016.66.

Torrence, C., and G. P. Compo, 1998: A practical guide to wavelet analysis. Bull. Amer. Meteor. Soc., 79, 61-78, https://doi.org/ 10.1175/1520-0477(1998)079<0061:APGTWA > 2.0.CO;2.

Vosper, S. B., 2015: Mountain waves and wakes generated by South Georgia: Implications for drag parametrization. Quart. J. Roy. Meteor. Soc., 141, 2813-2827, https://doi.org/10.1002/ qj.2566.

Warn-Varnas, A., J. Hawkins, P. Smolarkiewicz, S. Chin-Bing, D. King, and Z. Hallock, 2007: Solitary wave effects north of Strait of Messina. Ocean Modell., 18, 97-121, https://doi.org/ 10.1016/j.ocemod.2007.03.003.

Wedi, N. P., and P. K. Smolarkiewicz, 2004: Extending Gal-Chen and Somerville terrain-following coordinate transformation on time-dependent curvilinear boundaries. J. Comput. Phys., 193, 1-20, https://doi.org/10.1016/j.jcp.2003.07.034.

Wu, D. L., P. Preusse, S. D. Eckermann, J. H. Jiang, M. T. Juarez, L. Coy, and D. Y. Wang, 2006: Remote sounding of atmospheric gravity waves with satellite limb and nadir techniques. Adv. Space Res., 37, 2269-2277, https://doi.org/ 10.1016/j.asr.2005.07.031. 